\title{
Hemoglobin oxidation-dependent reactions promote interactions with band 3 and oxidative changes in sickle cell-derived microparticles
}

\author{
Sirsendu Jana, ${ }^{1}$ Michael Brad Strader, ${ }^{1}$ Fantao Meng, ${ }^{1}$ Wayne Hicks, ${ }^{1}$ Tigist Kassa, ${ }^{1}$ \\ Ivan Tarandovskiy, ${ }^{1}$ Silvia De Paoli, ${ }^{2}$ Jan Simak, ${ }^{2}$ Michael R. Heaven, ${ }^{3}$ John D. Belcher, ${ }^{4}$ \\ Gregory M. Vercellotti, ${ }^{4}$ and Abdu I. Alayash ${ }^{1}$ \\ 'Laboratory of Biochemistry and Vascular Biology and 'Laboratory of Cellular Hematology, Division of Blood Components \\ and Devices, Center for Biologics Evaluation and Research, FDA, Silver Spring, Maryland, USA. ${ }^{3}$ Vulcan Analytical LLC, \\ Birmingham, Alabama, USA. ${ }^{4}$ Division of Hematology, Oncology and Transplantation, University of Minnesota Medical \\ School, Minneapolis, Minnesota, USA.
}

The contribution of intracellular hemoglobin $(\mathrm{Hb})$ oxidation to RBC-derived microparticle (MP) formation is poorly defined in sickle cell disease (SCD). Here we report that sickle $\mathrm{Hb}$ (HbS) oxidation, coupled with changes in cytosolic antioxidative proteins, is associated with membrane alterations and MP formation in homozygous Townes-sickle cell (Townes-SS) mice. Photometric and proteomic analyses confirmed the presence of high levels of $\mathrm{Hb}$ oxidation intermediates (ferric/ferryl) and consequent $\beta$-globin posttranslational modifications, including the irreversible oxidation of $\beta$ Cys93 and the ubiquitination of $\beta$ Lys96 and $\beta$ Lys145. This is the first report to our knowledge to link the UPS (via ubiquitinated $\mathrm{Hb}$ and other proteins) to oxidative stress. Ferryl $\mathrm{Hb}$ also induced complex formation with band 3 and RBC membrane proteins. Incubation of Townes-SS MPs with human endothelial cells caused greater loss of monolayer integrity, apoptotic activation, heme oxygenase-1 induction, and concomitant bioenergetic imbalance compared with control Townes-AA MPs. MPs obtained from Townes-SS mice treated with hydroxyurea produced fewer posttranslational $\mathrm{Hb}$ modifications. In vitro, hydroxyurea reduced the levels of ferryl $\mathrm{Hb}$ and shielded its target residue, $\beta$ Суs93, by a process of S-nitrosylation. These mechanistic analyses suggest potential antioxidative therapeutic modalities that may interrupt MP heme-mediated pathophysiology in SCD patients.

Authorship note: SJ and MBS contributed equally to this work.

Conflict of interest: JDB and GMV receive research funding from $\mathrm{CSL}$ Behring.

License: Copyright 2018, American Society for Clinical Investigation.

Submitted: February 9, 2018 Accepted: September 13, 2018 Published: November 2, 2018

\section{Reference information:} JCI Insight. 2018;3(21):e120451. https://doi.org/10.1172/jci. insight.120451.

\section{Introduction}

Sickle cell disease (SCD) is characterized by chronic hemolysis, intermittent vaso-occlusive crises, and organ damage (1). Hemolysis results in the release of RBC-derived microparticles (MPs) and hemoglobin $(\mathrm{Hb})$ in circulation. It has previously been reported that heme released (approximately $25 \mu \mathrm{M}$ ) during a sickling crisis (originating from these 2 sources) may contribute to the complications of blood vessel injury and inflammation $(2,3)$. Several molecular mechanisms that contribute to a high oxidative burden in SCD patients have been proposed in recent years (4). Oxidative stress is increased in SCD and likely plays a significant role in the pathophysiology of SCD-related microvascular dysfunction, vaso-occlusion, and development of organ damage $(5,6)$. NADPH oxidase-derived ROS reported in sickle RBCs may also cause direct oxidative damage to a variety of subcellular structures, leading to a reduction in deformability that consequently increases $\mathrm{RBC}$ fragility and hemolysis $(6,7)$.

A number of recent preclinical models have provided considerable support to the notion that cell-free $\mathrm{Hb}$ (infused and/or released from outdated blood in normal or diseased animals) plays a significant role in the development of oxidative toxicity (8-10). In one report, oxidized $\mathrm{HbFe}^{3+}$ (metHb) or free heme was shown to trigger vaso-occlusion in SCD (but not in normal WT control) mice. The fact that metHb (but not cyanome$\mathrm{tHb}$, in which the iron is blocked by cyanide) induced vaso-occlusion indicated that heme liberation was necessary. In the same study, $\mathrm{Hb}$-induced vaso-occlusion was blocked by the metHb-reducing agent methylene blue, 
haptoglobin (Hp), and the heme-binding protein hemopexin (HPX) (8). Heme (released from Hb), now recognized by many as a damage-associated molecular pattern (DAMP) molecule, was shown to elicit vaso-occlusion (stasis) and acute chest syndrome in transgenic sickle mice by binding to endothelial TLR4 $(8,11)$.

The unique oxidative environment (within sickle cell RBCs [SS RBCs]) acts as an incubator for oxidized $\mathrm{Hb}$ redox forms (12). Because of the catalytic nature of these oxidation reactions, $\mathrm{Hb}$ is proposed to participate (inside and outside RBCs) in pseudoenzymatic radical side reactions (12). Moreover, we have recently reported that free sickle $\mathrm{Hb}(\mathrm{HbS})$ oxidizes faster, remains locked in a highly oxidizing ferryl state $\left(\mathrm{HbFe}^{4+}\right)$ longer, and loses its heme more rapidly than $\mathrm{HbA}$. In the same report, we also found that the persistent ferryl $\mathrm{HbS}$ (and its associated protein radicals) was responsible for irreversibly oxidizing $\mathrm{Hb}$ specific "hotspot" amino acids including $\beta$ Cys93, as well as targeting subcellular organelles such as the mitochondria (13).

Several MP subtypes have been observed in SCD blood, namely those derived from RBCs, platelets, endothelial cells, and monocytes. MPs increase in number as they bud from RBCs after cycles of sickling/ unsickling $(14,15)$. MPs are submicron, unilamellar vesicles possessing a lipid bilayer and proteins derived from the plasma membrane of their parent cells of origin. RBC-derived MPs (isolated from the plasma of freshly drawn blood) have been shown to contain all $\mathrm{Hb}$ components in a pattern like that of parent $\mathrm{RBCs}$ (14). Approximately $20 \%-30 \%$ of the RBC-derived MP content consists of $\mathrm{Hb}$; however, these vesicles also contain enzymes, microRNA (miRNA), and other multifunctional biologic mediators (15). In transgenic SCD mice, infusion of heme-laden MPs has been shown to trigger rapid vaso-occlusion in kidneys and compromise microvascular dilation ex vivo (16). These vascular effects were largely blocked for both in vitro and in vivo conditions by the heme-scavenging protein HPX and by the PS antagonist annexin V (3). This suggests that $\mathrm{Hb}$ oxidative pathways (which lead to heme loss) are likely occurring within circulating particles. There is therefore a need for the basic understanding of the molecular events (inside RBCs/MPs) that ultimately lead to $\mathrm{Hb} /$ heme loss to the circulation and their effect on the vascular system.

In this study, we monitored the kinetics of $\mathrm{Hb}$ oxidation as well as changes in the proteomic landscape of transgenic sickle (Townes-SS) mouse RBC-derived MPs. Specifically, this study focused on Hb pseudoperoxidative activity, which is responsible for driving RBC cytosolic and membrane changes and promoting oxidative toxicity toward the vascular system. We also compared the toxicities of RBC-derived MPs from Townes-SS mice with those from control Townes-AA mice on cultured human umbilical vein endothelial cells (HUVECs). Finally, we have revealed that MPs from Townes-SS mice treated with hydroxyurea (HU; one of 2 FDA-approved drugs for the treatment of SCD patients) reversed many oxidative changes within these vesicles. Interestingly, a newly approved L-glutamine has been shown to have antioxidant properties (17).

\section{Results}

Electrophoretic confirmation of SCD and the characterization of red cell-derived MPs from sickle cell mice. Electrophoretic mobility of hemolysates obtained from homozygous (SS), heterozygous (AS), and normal control (AA) Townes mice were carried out using isoelectric focusing to confirm the phenotype of blood samples derived from each animal (data not shown). RBC-derived MPs were then generated by shear stress from RBCs of Townes-AA or -SS mouse blood using a hypodermic syringe. The method used in this study was adopted with some modification from a previously published method using a cell disruptor (18), thus mimicking physiological fluid-shear stress that occurs within vessels during circulation. MP vesicular structure was confirmed by transmission electron microcopy (TEM). TEM images of MPs isolated from Townes-AA and -SS mouse RBCs are shown in Figure 1, A and B. Although an equal quantity of AA RBCs always had a lower yield of MPs compared with SS RBCs, AA and SS MPs appeared very similar under the TEM. Most MPs appeared as dense lipid vesicular structures between 100 and $300 \mathrm{~nm}$ in size. However, a few smaller MPs $(\sim 80 \mathrm{~nm})$ and larger vesicles $(>300 \mathrm{~nm})$ were also seen. Interestingly, some SS MPs also showed dense protein aggregates on the vesicular membrane and within the vesicles (Figure 1C). Flow cytometry was done using FITC-conjugated annexin V to count and confirm shear stress MPs (Figure 1D) bearing externalized phosphatidylserine (PS). Both AA and SS MPs showed a small variation in the number of PS-positive vesicles, ranging from $80 \%$ to $90 \%$ of the total population. For proteomic, biochemical, and spectroscopic analysis, MPs were compared after normalization to total protein content.

Spectrophotometric and chromatographic analyses of MPs reveal heme iron oxidation and subunit changes in sickle cell $\mathrm{Hb}$. To understand how RBCs' internal $\mathrm{Hb}$ oxidation reactions contribute to heme loss and membrane alterations, we monitored the progression of these reactions continuously for an extended 
A

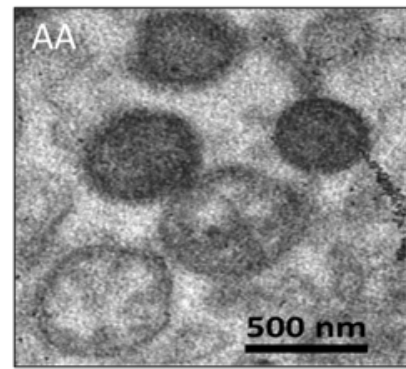

C

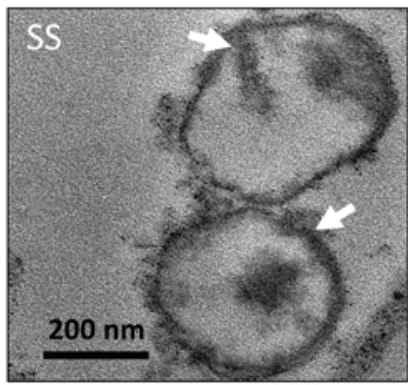

B

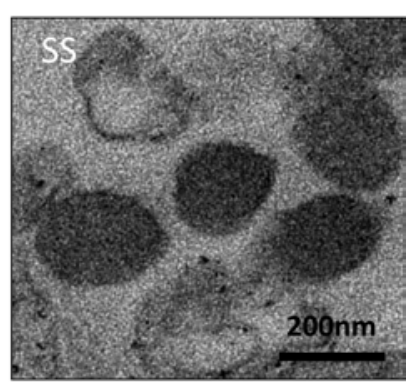

D

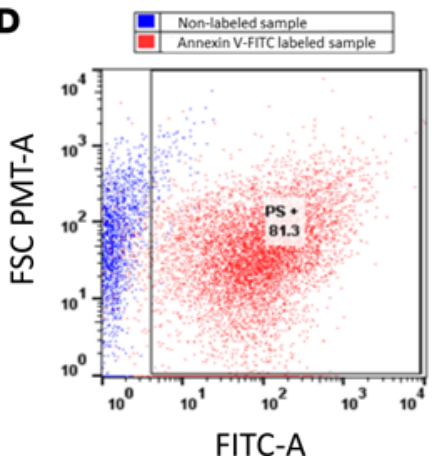

Figure 1. Characterization of RBC-derived microparticles from Townes mice. Representative transmission electron microscopic images showing Townes-AA (A) and Townes-SS RBC-derived (B and C) shear stress MPs of size ranging from 200 to 500 $\mathrm{nm}$. Solid white arrows indicate dense protein aggregates within MPs. All images are representative of experiments repeated at least 3 times and obtained from different MP preparations. (D) Representative scatter plot shows quantification of PS+ MPs by flow cytometry using FITC-conjugated annexin $V$ following calibration with standard silica beads. Scale bars: $500 \mathrm{~nm}$ (A); $200 \mathrm{~nm}$ (B and C).

period of 36 hours at room temperature using highly specialized spectrophotometric methods. These incubation times were considerably longer than the half-life of plasma circulating MPs in vivo $(14,15)$. A typical UV-visible absorbance spectrum captured during autoxidation (spontaneous) of free $\mathrm{HbA}$ is shown in Figure 2A. Ferrous $\mathrm{Hb}\left(\mathrm{HbFe}^{+2}\right)$ (2 major peaks, at 541 and $577 \mathrm{~nm}$ ) was slowly transformed into the ferric/met form $\left(\mathrm{HbFe}^{+3}\right)$ (absorbs strongly at $630 \mathrm{~nm}$ ). The progression of oxidation within MPs derived from Townes-SS blood is shown in Figure 2B. Similar spectral changes were observed in AA MP solutions due to the transformation of ferrous $\mathrm{Hb}$ in a classic pseudoperoxidative redox cycle to ferryl/ferric states. However, the initial oxidation rates for ferrous $\mathrm{Hb}$ were slightly faster in SS MP solutions $\left(K_{\text {auto }}=0.044\right.$ hours $^{-1}$ vs. $K_{\text {auto }}=0.027$ hours $\left.^{-1}\right)$ and there was less metHb accumulated in SS MPs (35\%) as compared with AA MPs (45\%) after 36-hour incubation (data not shown). The highly reactive ferryl $\mathrm{Hb}\left(\mathrm{HbFe}^{+4}\right)$ intermediate (peaks at 545 and $584 \mathrm{~nm}$ and a flattened region between 600 and $650 \mathrm{~nm}$ ) was apparent toward the end of the incubation period in both SS and, to lesser, extent AA MPs (data not shown for AA MPs). Ferryl $\mathrm{Hb}$ was also verified by adding sodium sulfide $\left(\mathrm{Na}_{2} \mathrm{~S}\right)$ to convert it to sulfhemoglobin (sulfHb), which absorbs strongly at 418 and $620 \mathrm{~nm}$, respectively. Due to solution turbidity and low levels of ferryl $\mathrm{Hb}$ in MPs, we monitored sulfHb formation in the Soret region $(418 \mathrm{~nm}$ ) (data not shown). These data strongly contrast with the cell-free HbA control, where minimal spectral changes were detected within the same time frame (Figure 2A).

When challenged with $\mathrm{H}_{2} \mathrm{O}_{2}$, free $\mathrm{HbS}$ undergoes oxidative changes in both $\alpha$ and $\beta$ subunits that involve irreversible oxidation of $\beta$ Cys93 and formation of heme-protein adducts $(19,20)$. These structural changes can be identified by reverse-phase HPLC methods and can be used as diagnostic biomarkers of oxidative changes in the protein and in blood (21). HPLC analysis of $\mathrm{HbS}$ subunits within MPs showed considerable alterations in both the $\beta$ and $\alpha$ subunits, likely due to the effects of ferryl $\mathrm{Hb}$-mediated oxidative reactions (22). AA MP samples revealed little or no modification of either the $\beta$ or $\alpha$ subunit (Figure 2C, top panel), which correlates well with the spectrophotometric $\mathrm{HbA}$ control data in Figure 2A. SS MP samples, however, showed a marked reduction in $\alpha$ - and $\beta^{\mathrm{S}}$-globin subunits (Figure $2 \mathrm{C}$, bottom panel), consistent with the $\mathrm{HbS}$ oxidation shown in Figure 2B. Heme peaks in both samples remained constant for all runs, which was used as an internal control to evaluate changes seen within the protein as a function of time (Figure $2 \mathrm{C}$, top and bottom panels). Biphasic time courses during $\mathrm{Hb}$ autoxidation within MPs were observed, with initial metHb levels of $30 \%-40 \%$; the levels then slightly declined in the first approximately 10 hours (likely due to the activity of RBCs' residual antioxidant enzymes within MPs), again reaching a maximum of $35 \%-40 \%$ of total $\mathrm{Hb}$ (Figure $2 \mathrm{D}$ ). There was a significant difference in metHb levels between the start and end point of the time course $(P=0.044$ for AA MPs vs. $P=$ 0.005 for SS MPs), and there was also a significant difference between the start and 10-hour time point 
A

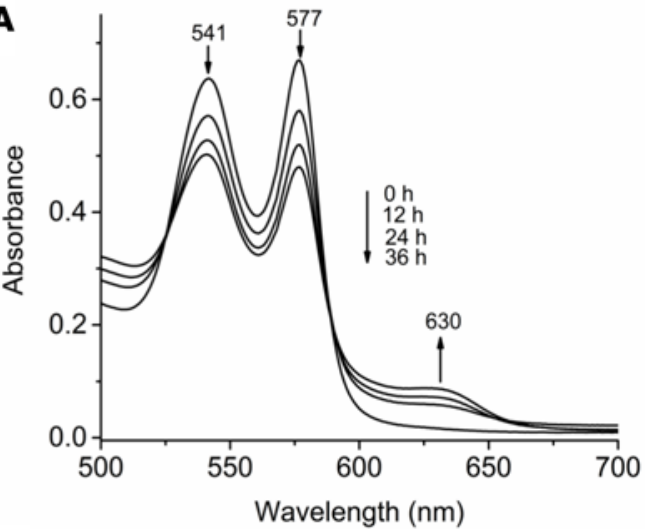

C

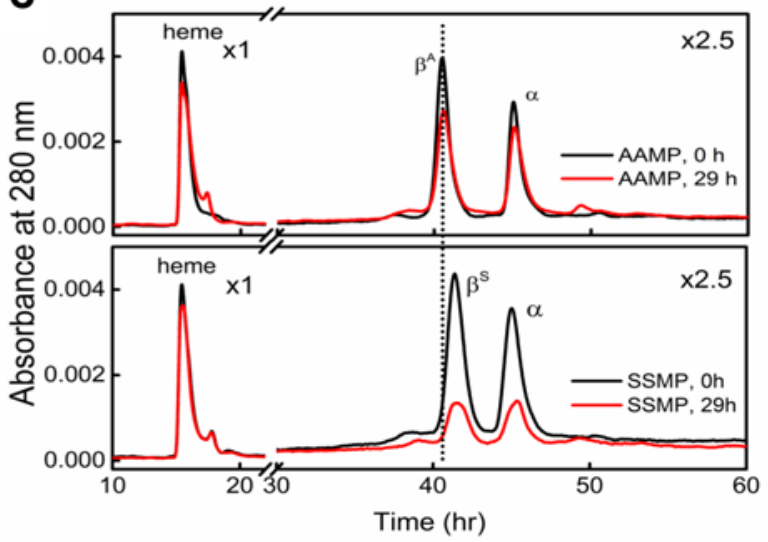

$\mathbf{E}$



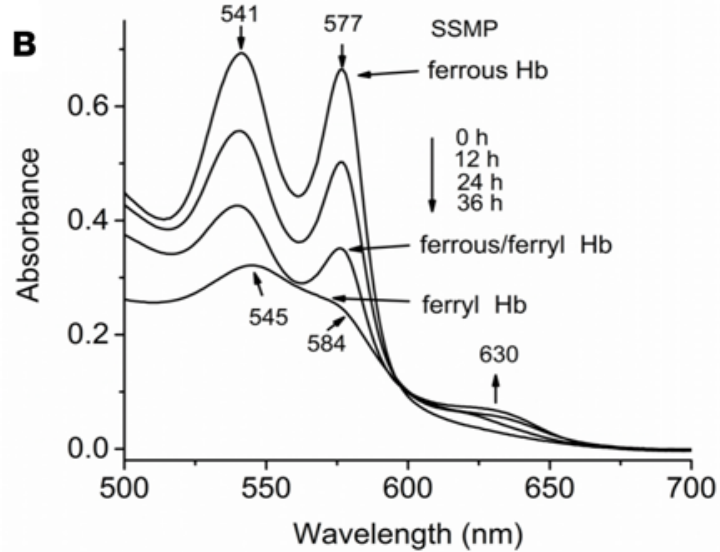

D

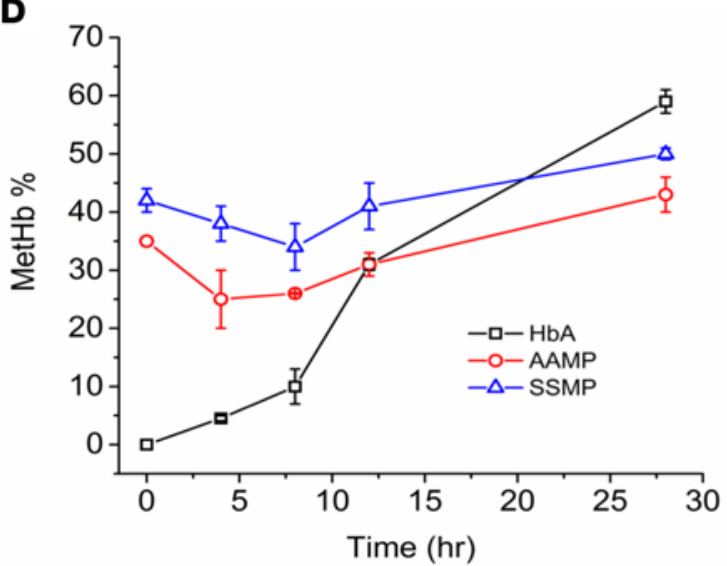

$\mathbf{F}$

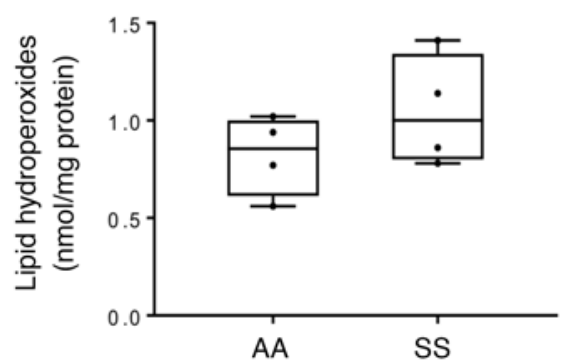

Figure 2. Hemoglobin S within microparticles undergoes oxidation and oxidative changes. Kinetic absorbance spectra of $\mathrm{HbA}$ control (A) and RBC MPs prepared from Townes-SS mice (B) (note: ferryl Hb spectrum is recognized by 2 new peaks at 545 and $584 \mathrm{~nm}$ and a flattened region between 500 and 700 $\mathrm{nm}$ ). The samples were incubated for the indicated times in PBS at $37^{\circ} \mathrm{C}$. Reverse-phase HPLC analyses of RBC MPs (AA and SS) before and after 36 hours of incubation (autoxidation) (C). The flow rate was $1 \mathrm{ml} / \mathrm{min}$ at $25^{\circ} \mathrm{C}$. The eluate was monitored at $280 \mathrm{~nm}$ (for globin chains) and $405 \mathrm{~nm}$ (for heme). (D) Time course kinetics of metHb formation during autoxidation of $\mathrm{HbA}$ and $\mathrm{HbS}$ inside MPs prepared from AA and SS mice compared with free $\mathrm{HbA}$ samples, as determined spectrophotometrically during the 30-hour incubation. (E) Carbonylated protein content and (F) total lipid hydroperoxide content were measured in RBC MPs $(n=4)$. Upper horizontal line in box plots represents 75 th percentile, lower horizontal line represents 25 th percentile, and horizontal line within box represents mean value. Vertical error bars represent $95 \%$ confidence interval. Student's $t$ test, 2-tailed, ${ }^{*} P<0.05$.

( $P=0.001$ for AA MP vs. $P=0.020$ for SS MP). Because of the presence of residual antioxidant enzymes (SOD and catalase) within MPs, the metHb levels inside these MPs were lower than that of free $\mathrm{Hb}(P=0.001$ for AA MP and $P=0.004$ for SS MP). This is contrasted with the linear kinetics of metHb accumulation (up to $65 \%-$ $75 \%$ ) in the free $\mathrm{HbA}$ solutions. We also carried out initial assessments of oxidative modification markers in the MPs, e.g., protein carbonylation and lipid hydroperoxide formation. A significantly higher level of carbonylated proteins in RBC MPs was obtained from Townes-SS mice compared with AA controls (Figure 2E). However, we did not see any significant difference in lipid hydroperoxide level between the 2 groups of MPs (Figure 2F). 
A Relative ion current intensity difference in antioxidant enzymes

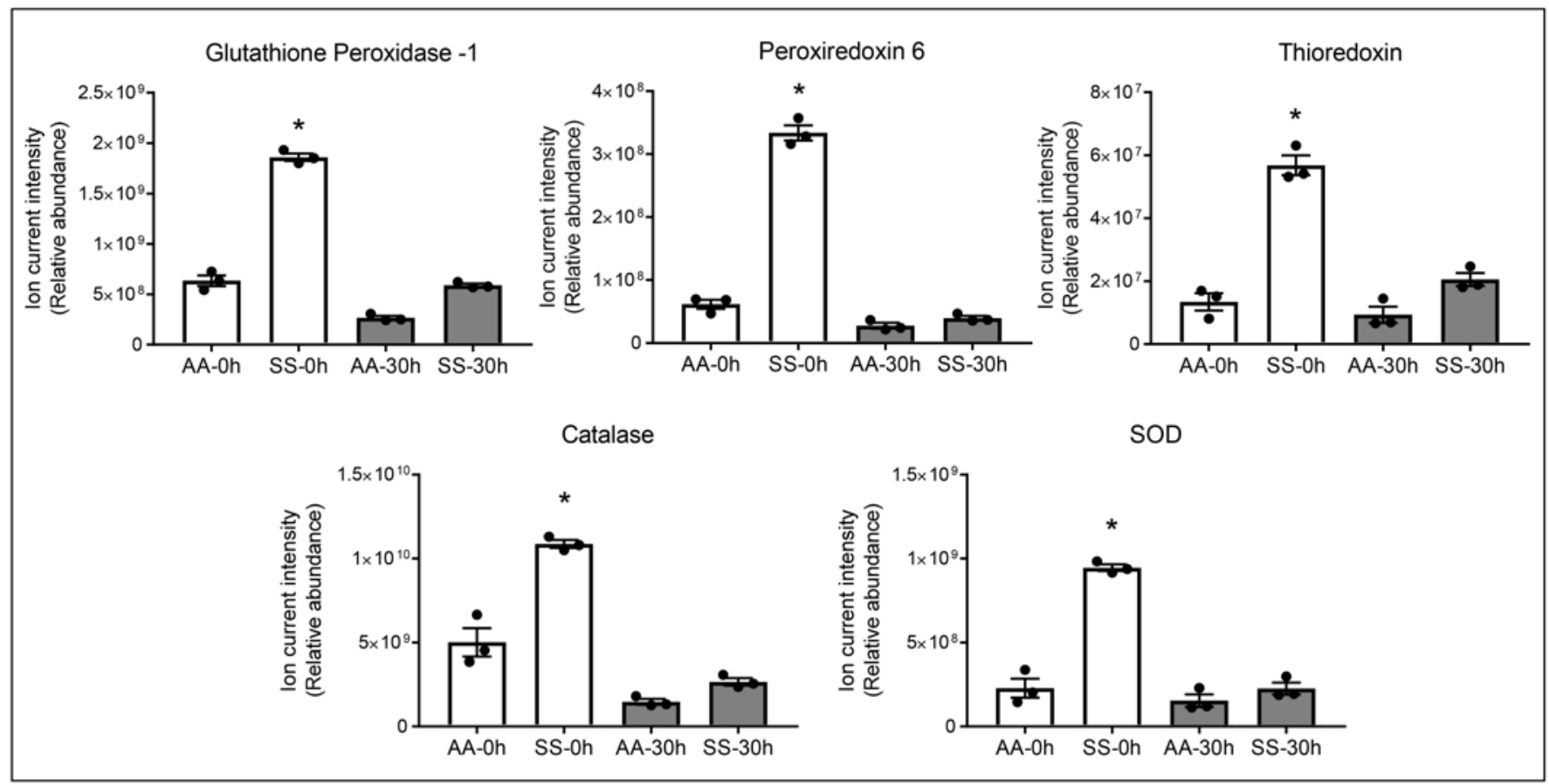



0 hours Sickle/Wild type mice fold-change $(\log 2)$

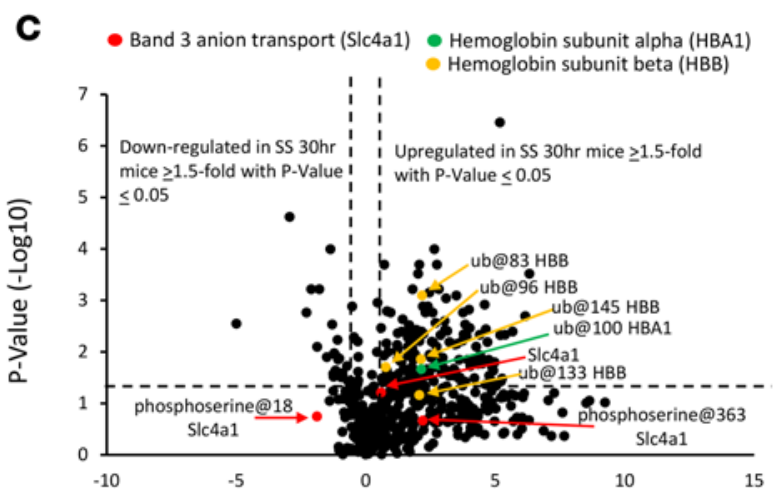

30 hours Sickle/Wild type mice fold-change (Log2)

Figure 3. Changes in antioxidative proteins parallel hemoglobin S oxidation in microparticles. (A) Individual plots represent mean \pm SEM of relative abundance determined from summed ion current intensity values for each oxidative enzyme, representing AA and SS microparticles ( $n=3$ ) at 0- and 30-hour incubations $\left(37^{\circ} \mathrm{C}\right)$; measurements were determined by peptide MS1 chromatogram intensities that were combined for each protein into protein-level data. (B and C) Volcano plots ( 0 and 30 hours) represent average relative fold differences determined by plotting $P$ values $\left(-\log _{10}\right)$ for each protein against the calculated fold change $\left(\log _{2}\right)$ difference (of that protein) in SS MP relative to AA MP peptide MS1 chromatogram intensities that were combined for each protein into protein-level data.ub@ and phosphoserine@ refer to the posttranslational modifications ubiquitination and serine phosphorylation (at the specified amino acid position), respectively. All peptide-spectrum matches were obtained using a strict $1 \%$ protein FDR. $P$ values were generated by applying a $t$ test statistic to $\log _{2}$-transformed fold change values for each mouse biological condition. All points (for both volcano plots) on or above the horizontal line parallel to the $x$ axis represent proteins with $P$ values of 0.05 or lower. All points on or to the left of the first vertical line parallel to the $y$ axis represent proteins that were downregulated in SS mice 1.5-fold or greater. All points on or to the right of the second vertical line parallel to the $y$ axis represent proteins that were upregulated in SS mice 1.5-fold or greater. Bars represent average mean value; each dot in the bars represents an individual data point, and vertical error bars represent SEM. Student's $t$ test, 2 -tailed, ${ }^{*} P<0.05$ vs. corresponding AA 0 -hour samples.

Changes in MP antioxidative proteins occur in parallel to $\mathrm{Hb}$ oxidation. We used a high-resolution accurate mass (HRAM) mass spectrometry (HRAM-MS) strategy to quantify proteomic changes within RBC MPs from AA and SS mouse samples at $t=0$ and 30 hours. As shown in Figure 3A, a considerable reduction in the antioxidative enzyme/protein levels (glutathione peroxidase 1, peroxiredoxin 6 , thioredoxin, catalase, SOD) in SS mice at 30 hours was observed, which largely correlated with the $\mathrm{Hb}$ 



Figure 4. Mass spectrometric analysis of microparticles from sickle mice reveals amino acid posttranslational modifications. MS/MS fragmentation spectra representing (A) y and b fragment ions matched to the Hb tryptic peptide GTFATSELHCDKLHVDPENFR (residues 83-104) containing cysteic acid (48 Da) at $\beta$ Cys93.

(B) y and b ions matched to the Hb tryptic peptide GTFATSELHCDKLHVDPENFR containing the ubiquitin diglycine signature modification (114 Da) at $\beta$ Lys96. (C) y and b ions matched to the $\mathrm{Hb}$ tryptic peptide VVACVANALAHKYHK (residues 335133-335145) containing the ubiquitin diglycine signature modification (114 Da) at $\beta$ Lys145.

oxidation kinetics shown in Figure 2D. The substantial reduction of these enzymes after 30 hours in SS mice suggests that these proteins were consumed in the process of counteracting the rising levels of $\mathrm{Hb}$ oxidation products including ferric/ferryl Hbs. This is consistent with reported redox imbalances and impairments in enzymes involved in antioxidant protection and elimination of $\mathrm{H}_{2} \mathrm{O}_{2}$ within SS RBCs (4, 23). Also, of note, the levels of antioxidant enzymes were higher in SS MPs compared with AA MPs at both time points which may reflect the fact that SS RBCs were experiencing more oxidative stress and consequently higher levels of oxidized $\mathrm{Hb}$ at $t=0$ (Figure 2D). 



\section{Circulating MPs}
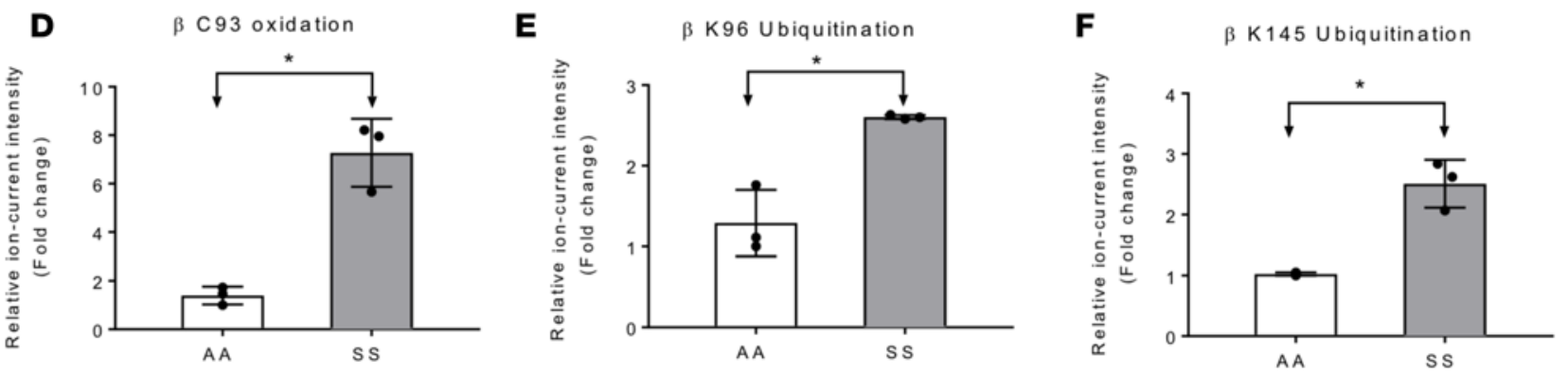

G

\section{Ubiquitin}

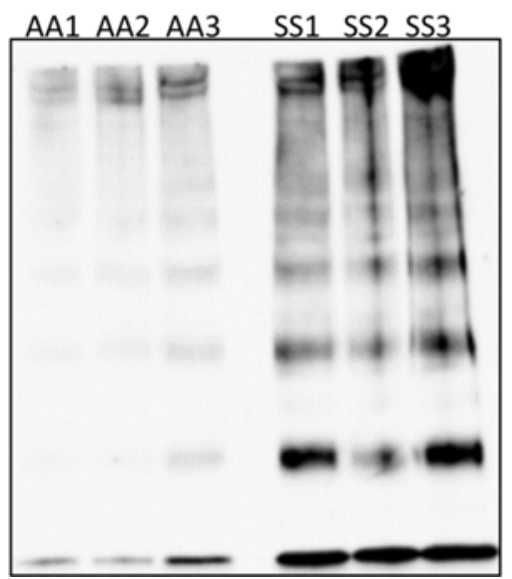

H

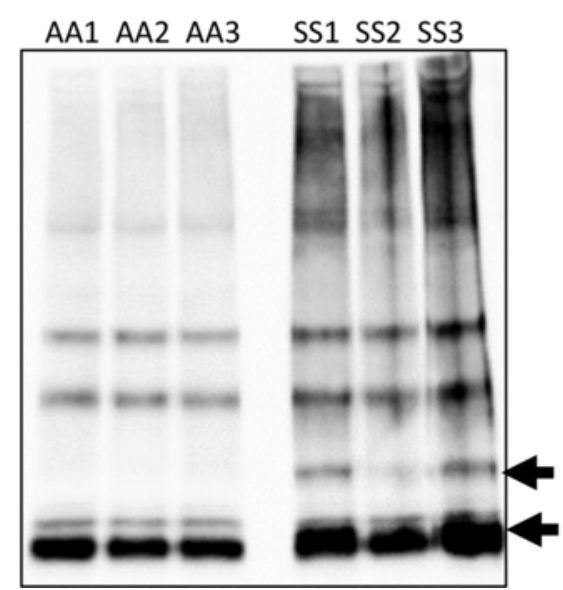

I SDS-PAGE

AA1 AA2 AA3 SS1 SS2 SS3



Figure 5. Representations of amino acid posttranslational modifications reveal significant changes in both shear stress and circulating microparticles. MS/MS fragmentation spectra were used to search for posttranslational modifications (PTMs) previously known to be associated with $\mathrm{Hb}$ oxidative stress. (A-F) Relative ion current intensity fold changes corresponding to each $\mathrm{Hb}$ amino acid-specific PTM from AA and SS mouse shear stress and circulating microparticles $(n=3)$; measurements were determined by Hb peptide MS1 chromatogram intensities for each PTM. All peptide-spectrum matches were obtained using a strict 1\% protein FDR. (G) Ubiquitinated proteins in AA and SS RBC lysates from 3 sets of mice were immunoprecipitated by protein $\mathrm{G}$ agarose beads using anti-ubiquitin antibody. (H) Ubiquitin bound $\mathrm{Hb}$ complex formation was analyzed using anti- $\mathrm{Hb} \beta$ antibody. (I) Proteins present in the eluted immunoprecipitate were also resolved by SDS-PACE and stained with Coomassie blue. Arrows represent $\mathrm{Hb} \beta$ subunit. Bars represent average mean value; each dot in the bars represents an individual data point; and vertical error bars represent SEM. Student's $t$ test, 2-tailed, ${ }^{*} P<0.05(n=3)$.

$\mathrm{Hb}$ undergoes posttranslational modification within MPs. A comprehensive proteomic comparison of AA and SS mouse MPs at $t=0$ and $t=30$ hours is shown in Figure 3, B and C - by proteome-wide quantification volcano plots consisting of $P$ values $\left(-\log _{10}\right)$ of each protein versus fold change $\left(\log _{2}\right)$ difference (of that protein) in SS compared with AA MPs - to visually show how abundant proteins were distributed between the $2 \mathrm{MP}$ populations (see Supplemental Tables 1 and 2; supplemental material available online with this article; https://doi.org/10.1172/jci.insight.120451DS1). To generate data for these plots, 
we applied liquid chromatography-tandem MS (LC-MS/MS) analysis on tryptic digests, and quantified peptides and proteins from each sample by high-resolution survey scan MS1 intensity values. For example, at $t=0$ this method allowed us to quantify 905 proteins across AA and SS mouse samples; 165 of these proteins were significantly more abundant $(\geq 1.5$-fold value and $P \leq 0.05)$ in the SS mouse proteome. Among the upregulated proteins in the SS mouse proteome were those associated with oxidative stress, including $\mathrm{Hb}$ antioxidant enzymes (described above), membrane structural proteins, mitochondrial proteins, heat shock proteins, and proteins involved in the ubiquitin proteasome machinery. Indeed, this trend was reproduced in additional SS mouse MP studies that included RBC lysate (see below for comparative data). We also included a stress associated posttranslational modification (PTM) analysis to search for PTMs associated with stress, including those associated with $\mathrm{Hb}$ oxidative modifications (ubiquitination, phosphorylation, cysteine trioxidation) $(13,24)$. Representative MS/MS fragmentation spectra are shown in Figure 4, A-C, for each PTM identified in the $\beta$ subunit of $\mathrm{HbS}$. From these searches, we observed in $\mathrm{SS}$ mice (shear stress and circulating) that $\mathrm{HbS}$ contained higher levels of irreversible $\beta \mathrm{Cys} 93$ oxidation as well as $\beta$ Lys96 and $\beta$ Lys145 ubiquitination compared with HbA from AA mouse MPs. $\beta$ Cys93 oxidation was $>2$-fold higher in shear stress-generated SS MPs (Figure 5A) and >4-fold higher in circulating SS MPs (Figure 5D). Irreversible oxidation of $\beta C y s 93$ has been shown by our group to be one of the prominent determining features in $\mathrm{HbS}$ oxidative instability. Furthermore, we have demonstrated that shielding this amino acid from oxidative challenges improves stability and gelation time $(25,26)$.

A remarkable finding in our PTM search was the discovery of ubiquitination at $\beta$ Lys 96 and $\beta$ Lys 145 (Figure 5, B, C, E, and F). To the best of our knowledge, this is the first report showing ubiquitination of $\mathrm{HbS}$ possibly due to accumulation of oxidatively damaged $\mathrm{Hb}$ molecules in RBCs/MPs. In addition to mass spectrometric identification, we were also able to capture ubiquitinated $\mathrm{Hb}$ within sickle $\mathrm{RBC}$ sollowing inhibition of the UPS and deubiquitinating enzymes (DUBs) by MG132 and N-ethylmalemide (NEM), respectively. Specifically, we employed an orthogonal co-immunoprecipitation (co-IP) assay using an anti-ubiquitin antibody (Figure 5, G and $\mathrm{H}$ ) to pull down ubiquitin-protein complexes. The immunoblot using an anti- $\mathrm{Hb} \beta$ antibody (Figure 5H) and Coomassie staining of SDS-PAGE gel (Figure 5I) of eluted ubiquitin-protein complexes from protein $\mathrm{G}$ agarose show higher levels of $\mathrm{Hb}$ monomers and dimers (indicated by black arrows) in SS mouse RBCs, confirming our results obtained from proteomic analysis. Taken together, our results suggest a relationship between oxidation of $\beta$ Cys 93 and the ubiquitination of $\beta$ Lys 96 and $\beta$ Lys 145 and the possibility of proteasomal inhibition resulting from redox imbalance within sickle RBCs.

Ferryl HbS forms a complex through a covalent attachment to RBC ghost membranes. RBC band 3 proteins have been shown to have increased tyrosine phosphorylation in patients with hemoglobinopathies including SCD and are also known to bind with oxidized forms of $\mathrm{Hb}$ to form complex (27-29). We therefore analyzed mouse RBC-derived MPs for possible band 3-Hb association by co-IP assay using a specific band 3 antibody. To investigate the extent of binding with different $\mathrm{Hb}$ oxidation intermediates and consequent $\mathrm{RBC}$ membrane alteration, we examined the reactivity of ferryl $\mathrm{Hb}$ and other redox forms (prepared by the reaction of ferric $\mathrm{Hb}$ with $\mathrm{H}_{2} \mathrm{O}_{2}$ ) toward human $\mathrm{RBC}$ ghost membranes. Figure 6A shows the absorbance spectra of freshly prepare $\mathrm{HbA}$ oxidation intermediates used in these experiments. Using ghost $\mathrm{RBC}$ membranes that were treated with different $\mathrm{Hb}$ redox states $\left(\mathrm{Fe}^{2+}, \mathrm{Fe}^{3+}\right.$, and $\left.\mathrm{Fe}^{4+}\right)$, we determined $\mathrm{Hb}$ intermediate binding to the RBC membrane by a similar co-IP assay (as done before with MPs) using an anti-band 3 antibody. Probing with an antibody against $\mathrm{Hb} \alpha$ chain following transfer of eluted proteins from immunoprecipitated band 3 to nitrocellulose membrane revealed binding of $\mathrm{Hb}$ with band 3 proteins. As shown in Figure $6 \mathrm{~B}$, ferrous and ferric $\mathrm{Hb}$ exposure did not cause any complex formation relative to untreated ghost membranes. However, an increased complex formation with $\mathrm{Hb}$ was seen in the band 3 eluates from membranes treated with ferryl protein (lane 4, Figure 6B). Proteins eluted from band 3 immunoprecipitates were then resolved on SDS-PAGE. Coomassie staining of the immunoprecipitated band 3 eluates also revealed a high degree of complex formation by ferryl $\mathrm{Hb}$ (Figure 6C), as also seen by Western blotting. For quantitation of band intensities, densitometric image analysis was performed (Figure 6D) from a chosen area in the gel (red box) where significant band 3 accumulation was also noted (see Figure 6B, upper panel). Relative band intensities from the $120-\mathrm{kDa}$ region (red box) show a significant increase in complex formation by ferryl $\mathrm{Hb}$ (Figure 6D). To identify other proteins, present in the band $3-\mathrm{Hb}$ complex, we analyzed the eluted band $3 \mathrm{immu}-$ noprecipitates by MS. Proteomic analysis of bands in the SDS-PAGE gel revealed complex formation with several $\mathrm{RBC}$ membrane proteins and $\mathrm{Hb}$ subunits. Protein complexes extracted from ghost $\mathrm{RBC}$ membrane (treated with different $\mathrm{Hb}$ redox forms, specifically the ferryl form) contained several membrane-associated 
A
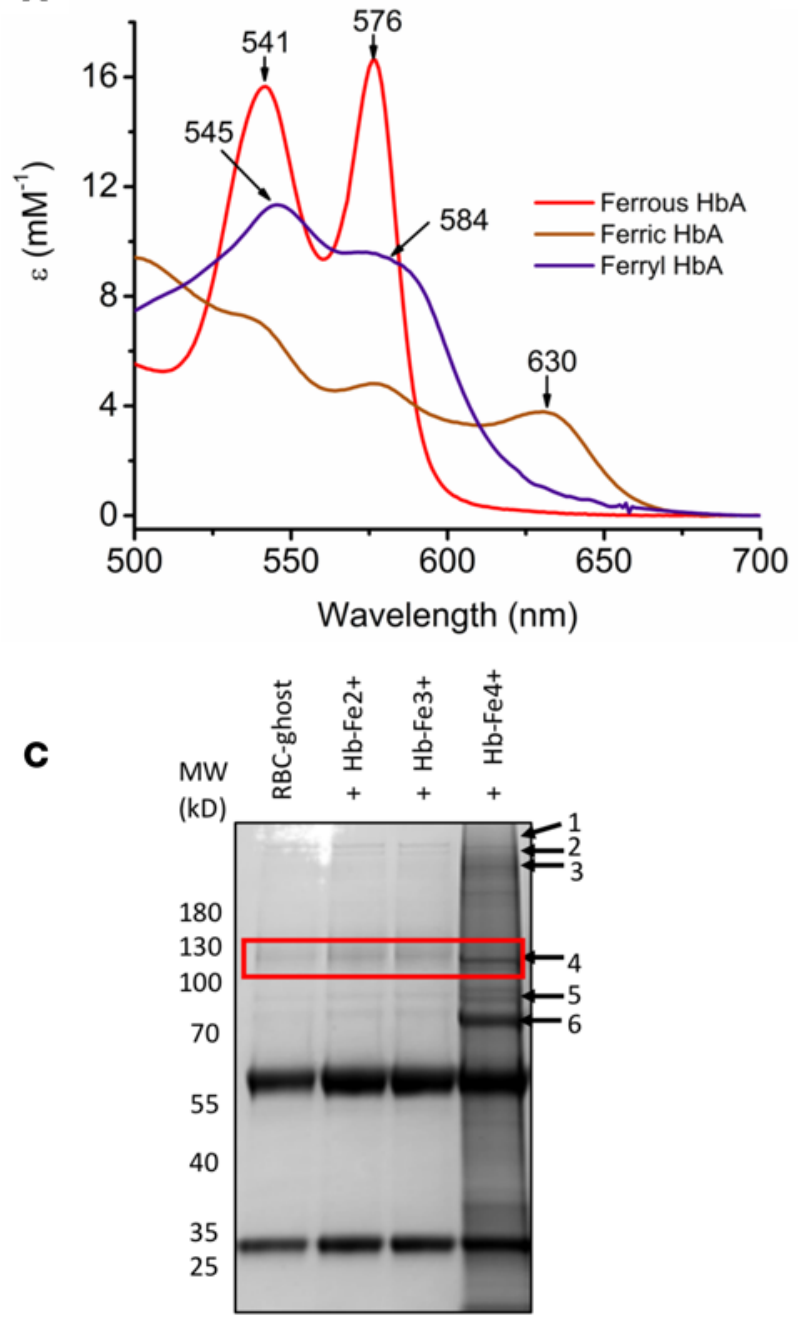


Figure 6. Hemoglobin oxidation promotes complex formation with band 3. Co-IP of band 3 was done and probed with anti-Hb antibody. (A) The absorbance spectra for the ferrous, ferric, and ferryl Hbs used in experiments. (B) Band 3-Hb complex formation in RBC ghost membrane was analyzed following in vitro incubation with different $\mathrm{Hb}$ oxidation states using a co-IP assay and immunoblotted using anti-band 3 and anti-Hb antibodies (lower panel). Upper panel: 90-kDa bands show equal band 3 loading; lower panel: Hb binding developed with anti-Hb antibody. Upper panel: 120-kDa bands indicate high molecular complex with band 3. (C) Eluted proteins from band 3 immunoprecipitates from RBC ghost membranes treated with or without $\mathrm{Hb}$ of different oxidation states were resolved by SDS-PACE, followed by staining with Coomassie blue. The gel bands were further analyzed by LC-MS. Higher levels of membrane protein complexes were found in ferryl $\mathrm{Hb}$-treated RBC ghost membranes (some of the major complexed proteins with $\mathrm{Hb} \alpha$ or $\beta$ subunits include [1] spectrin $\alpha$; [2] spectrin $\beta$, spectrin $\alpha, \mathrm{Hb} \beta$, band 3 anion transport protein, $\mathrm{Hb} \alpha$, ankyrin $1 ;$ [3] spectrin $\beta$ isoform $A, H b \beta$, band 3 anion transport protein, $\mathrm{Hb} \alpha$, ankyrin 1; [4] band 3 anion transport protein, ankyrin 1; [5] erythrocyte membrane protein band 4.2, $\mathrm{Hb} \beta$ subunit, band 3 anion transport protein, $\mathrm{Hb} \alpha$ subunit; [6] $\mathrm{Hb} \beta$, and band 3 anion transport protein). All proteins were searched using Mascot and listed in order of Mascot score. (D) Plot representing relative band intensities measured from a specific area of Coomassie-stained gel (120-kDa region, denoted by the red box in C). The values in the graph represent average mean value of 3 independent observations, and each dot in the bars represents individual data points. Vertical error bars represent SEM. Student's $t$ test, 2 -tailed, ${ }^{*} P<0.05$ vs. control $(n=3)$.

proteins, including spectrin, ankyrin, $\mathrm{Hb}$ (both $\alpha$ and $\beta$ subunits), and the band 3 anion transport protein (Figure 6C). More quantitative analysis is currently underway to fully analyze the nature of interactions between $\mathrm{Hb}$ and the $\mathrm{RBC}$ proteins.

MPs from SS mice influence vascular integrity and mitochondrial function. RBC-derived MPs from SCD patients carry a significant portion of heme and cell-free $\mathrm{Hb}$ that are delivered to vascular endothelial cells, causing oxidative stress, apoptosis, and vaso-occlusion (3). To investigate the interactions and associated toxicities of shear stress RBC MPs from Townes-SS versus -AA mice in human vascular endothelial cells, we used HUVECs that were exposed to RBC-derived MPs for varying periods of time (up to 12 hours). MPs are 
known to adhere efficiently to endothelial cells by altering expression of adhesion molecules $(3,15)$. We first monitored the expression of heme oxygenase-1 (HO-1) in HUVECs as an indicator of Hb cellular uptake of $\mathrm{Hb}$ or heme from MPs. Coincubation of SS MPs for 12 hours caused a significant rise in HO-1 level in HUVECs compared with AA MPs (Figure 7, A and B). Next, we sought to observe any changes in vascular endothelial membrane integrity following exposure to RBC-derived MPs by monitoring the permeability of high-molecular-weight dextran using Transwell inserts and by fluorescence microscopy. We first assessed the loss of endothelial monolayer integrity by monitoring the passage of FITC-conjugated dextran (40 kDa). Following 12 hours of incubation, Townes-SS MPs caused an almost 2-fold increase in dextran passage compared with corresponding AA MPs, which suggests a loss of monolayer integrity of HUVECs (Figure 7C). Fluorescence microscopy also revealed changes in the endothelial membrane adherens junctions, as indicated by a significantly altered pattern of the endothelial membrane-specific protein VE-cadherin following exposure to SS MPs for 12 hours (Figure 7D, right panel). To assess the loss of cell viability and apoptotic activation in MP-treated HUVECs, we determined the number of apoptotic cells by automated cell counting using annexin V-propidium iodide dual staining. We found a significantly higher number of apoptotic cells in SS MP-treated HUVECs compared with untreated cells (Figure 7E). However, AA MPs showed only a marginal increase in apoptotic cells relative to untreated control.

Since endothelial cells are dependent primarily on glycolytic energy production and partially on mitochondrial oxidative phosphorylation; we employed an extracellular flux analyzer (XF assay) to assess any impairment in energy metabolism. We monitored mitochondrial respiration and glycolytic activity in real time within AA and SS MP-treated HUVECs by measuring the mitochondrial oxygen consumption rate (OCR) and the extracellular acidification rate (ECAR), respectively. Representative OCR and ECAR plots are shown in Figure 7, F and G. Calculations of bioenergetic parameters from average OCR changes data did not reveal any alteration in basal mitochondrial OCR following MP treatments, when baseline respiration was corrected for any residual nonmitochondrial oxygen consumption (OCR after addition of rotenone/ antimycin A) (Figure $7 \mathrm{H}$ ). Interestingly, mitochondrial spare respiratory capacity or reserve capacity was marginally affected in HUVECs exposed to SS MPs, indicating compromised ability of the cells to respond to increased energy demand (Figure 7I). However, endothelial glycolytic capacity, which is the maximum ECAR achieved following oligomycin injection, was mostly unaffected by any of the MPs (data not shown).

$H U$ treatment reduces $H b$ posttranslational modifications. We repeated the same proteomic comparative approach (see Figure 3, B and C) on MPs derived from blood and RBC lysates with a new set of AA and SS mice $(n=3)$. In addition, we also treated SS mice with the anti-sickling agent HU for 10 days and analyzed MPs from HU-treated versus untreated SS mice. Expressing human $\mathrm{HbS}$ in SS mice reproducibly induced oxidative stress; this can be seen by comparing Figure 8A with the volcano plots represented in Figure 3. A total of 855 proteins across both SS MP and AA MP samples were identified; 276 of these proteins were significantly more abundant $(\geq 1.5$-fold value and $P \leq 0.05)$ in the SS mouse proteome. Once again, a majority of these abundant proteins were clearly associated with oxidative stress, including antioxidant enzymes, membrane structural proteins, heat shock proteins, and the $26 \mathrm{~S}$ proteasome, etc. (see Supplemental Table 3). As seen for Figure 3, a substantial increase in the abundance of particular mitochondrial proteins in SS relative to AA mouse MPs was also shown in the proteome-wide quantification volcano plot in Figure 8A. The presence of relatively abundant mitochondrial proteins in SS RBC/MP lysates is surprising. However, recent literature shows that excessive ROS in SCD are associated with abnormal mitochondrial retention $(30,31)$. The greater abundance of mitochondrial proteins and some antioxidants in SS MPs may also originate from immature RBCs (reticulocytes), as blood of Townes-SS mice contains a substantial population of reticulocytes (32). We recently reported that Townes-SS mice have $37 \%-58 \%$ reticulocytes as a percentage of total RBCs (33). As a follow-up, we performed an additional proteomic comparison of AA and SS samples for the RBC lysate (see Figure 8, A and B). There are some important differences between the MP and RBC lysate proteomes (see Supplemental Table 4). First, the SS RBC lysate contains substantially fewer mitochondrial proteins than the SS MP, which suggests that mitochondrial retention may be localized to MPs or alternatively mitochondrial proteins are enriched (along with other organellar proteins associated with reticulocytes) in the MP preparation. Second, the 5 enzymes listed in Figure $3 \mathrm{~A}$ (glutathione peroxidase 1, peroxiredoxin 6, thioredoxin, catalase, and SOD) were at comparable levels in AA and SS RBC lysates, which contrasts with the MP proteome. Upregulated proteins in the RBC lysate proteome consists primarily of cytosolic proteins including ribosomal proteins, translational proteins, cytoskeletal proteins, metabolic proteins, heat shock proteins, and ubiquitin proteasome machinery proteins. 
A

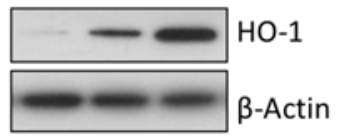

HUVEC AA SS

MP MP
B

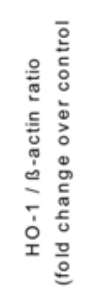

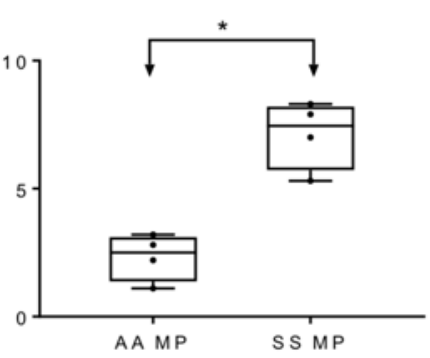

C

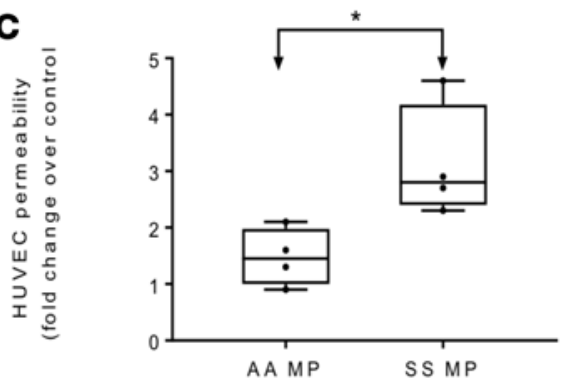

D

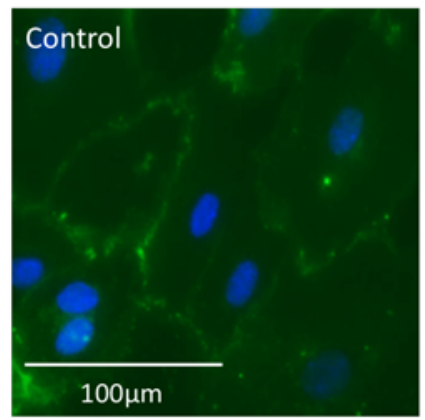

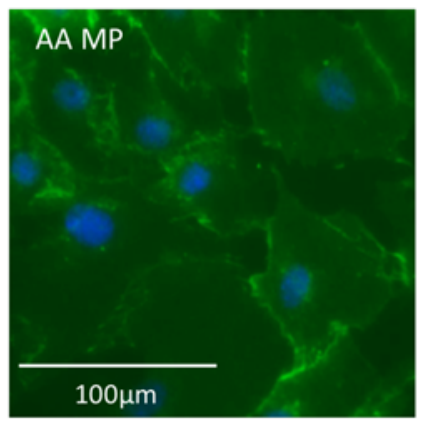

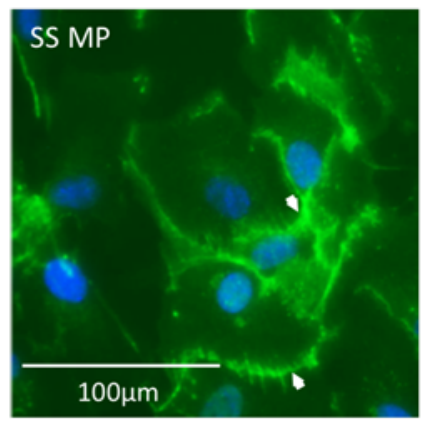

E

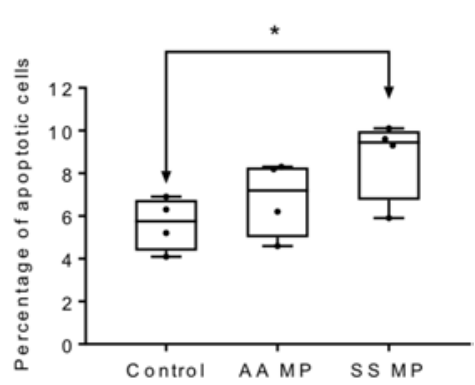

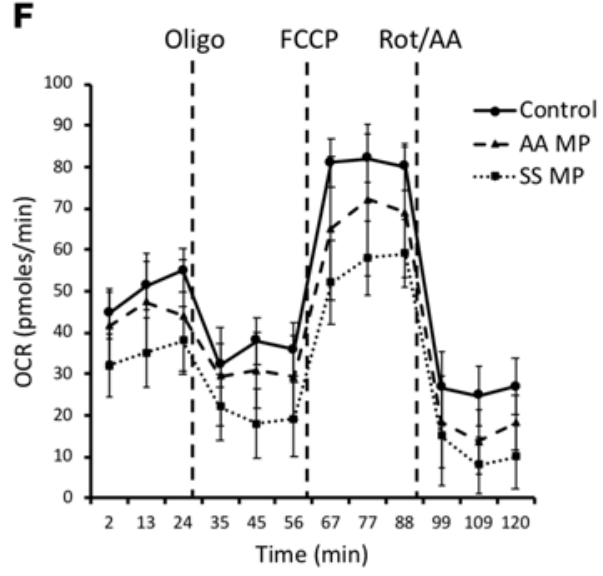

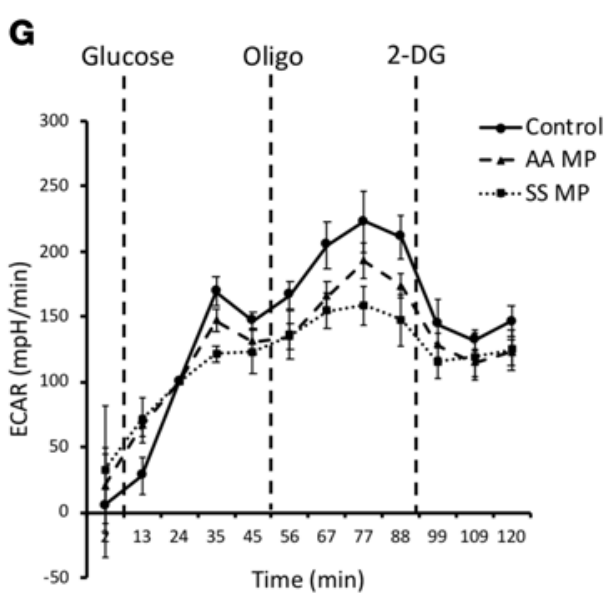

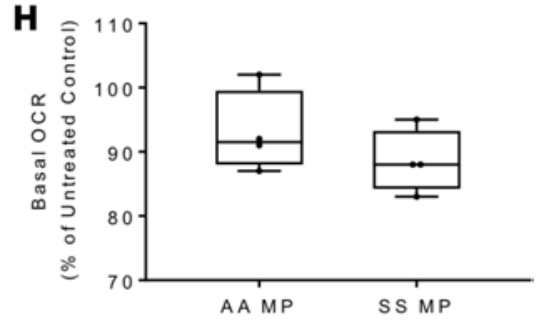

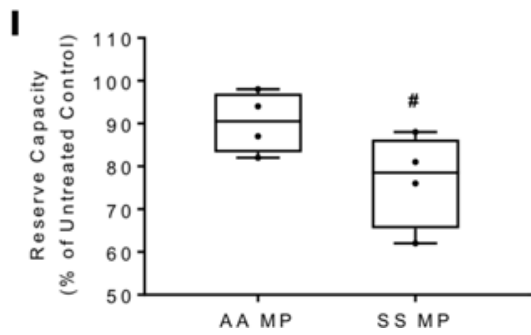

Figure 7. Townes-SS RBC-derived microparticles promote toxicity in human endothelial cells. (A) Oxidative toxicity in human umbilical vein endothelial cells (HUVECs) was assessed by expression of HO-1 protein. (B) Relative band intensity ratio representing expression of HO-1 normalized to corresponding $\beta$-actin level was measured using Bio-Rad Image Lab software $(n=4)(C)$ Loss of monolayer integrity in HUVECs exposed to Townes-AA or -SS RBC MPs for 12 hours, measured by passage of FITC-dextran through Transwell membranes $(n=4)$. (D) Representative fluorescence microscopic images of HUVEC endothelial membrane stained with anti-VE-cadherin (green) showing disruption of membrane integrity by SS MP treatment for 12 hours. (E) Number of apoptotic cells as quantified in HUVEC culture following 12 hours of incubation with SS or AA MPs by cell counter using annexin $V$ and propidium iodide $(n=6)$. ( $F$ and $\mathbf{G})$ Representative plots showing oxygen consumption rate (OCR) and extracellular acidification rate (ECAR) profiles in HUVECs obtained from extracellular flux analyzer following coincubation with either media (Control), Townes-AA MPs, or Townes-SS MPs for 12 hours. Data points represent average OCR/ECAR values of 6 similar wells. Following incubation with MPs, HUVEC were subjected to sequential automated injections of the ATP synthase blocker oligomycin (Oligo), oxidative phosphorylation uncoupler carbonyl cyanide 4-(trifluoromethoxy) phenylhydrazone (FCCP), and a cocktail of the mitochondrial respiration inhibitors rotenone and antimycin $A$ (AA) to obtain the full OCR profile. For ECAR profile, a sequential injections of glucose, oligomycin (Oligo), 2-deoxy-D-glucose (2DC) were made. (H) Average basal OCR and (I) mitochondrial reserve capacity were calculated from 4 separate OCR plots $(n=4) . \# P<0.05$ vs. control. Upper horizontal line in box plots represent 75 th percentile, lower horizontal line in represent 25 th percentile, horizontal line within box represents mean value. Vertical error bars represent $95 \%$ confidence interval. Student's $t$ test, 2 -tailed, ${ }^{*} P<0.05$. 


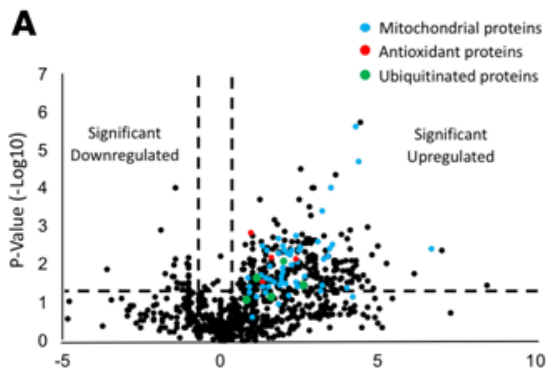

SS / AA mice (Log2 Fold-change)

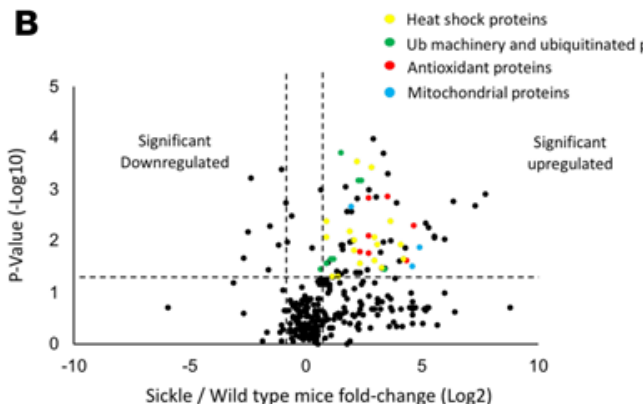

Sickle / Wild type mice fold-change (Log2)

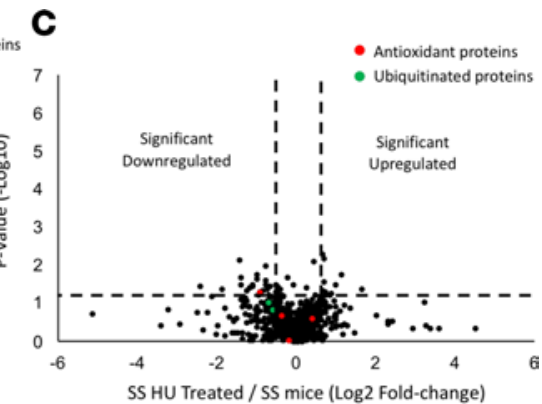

G
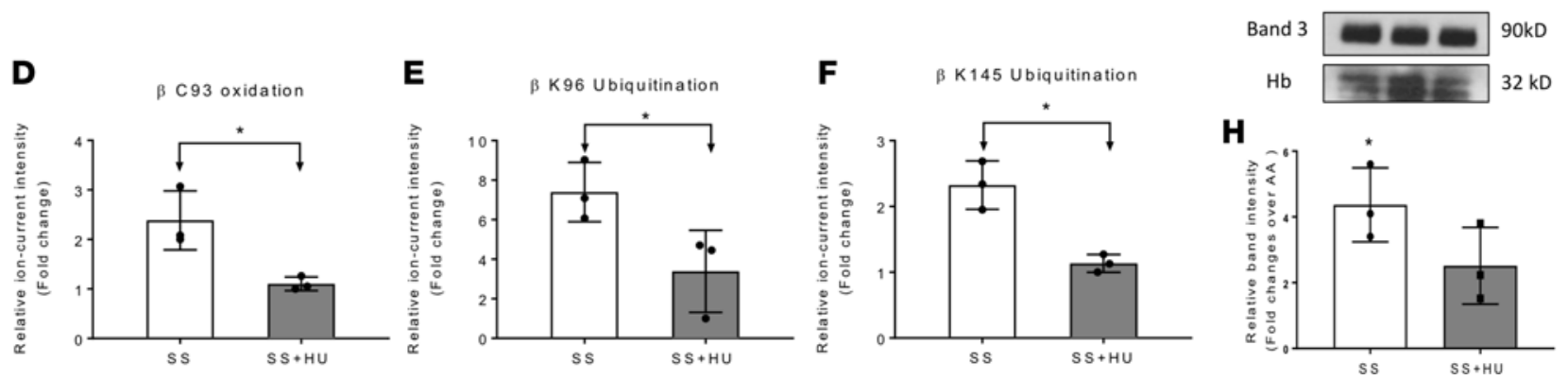

Figure 8. Hydroxyurea treatment reduces posttranslational modifications in microparticles. A second proteomic comparative analysis including MPs derived from the blood of a set of AA, SS, and hydroxyurea-treated (HU-treated) SS mice $(n=3)$. (A) Volcano plots represent average relative fold differences determined by plotting $P$ values $\left(-\log _{10}\right)$ for each protein against the calculated fold change $\left(\log _{2}\right)$ difference (of that protein) in SS relative to AA microparticle peptide MS1 chromatogram intensities that were combined for each protein into protein-level data. (B) Volcano plots represent average relative fold differences determined by plotting $P$ values $\left(-\log _{10}\right)$ for each protein against the calculated fold change ( $\log _{2}$ ) difference (of that protein) in SS relative to AA RBC lysate peptide MS1 chromatogram intensities that were combined for each protein into protein-level data. (C) Volcano plots represent average relative fold differences determined by plotting $P$ values $\left(-\log _{10}\right)$ for each protein against the calculated fold change (log $)$ difference (of that protein) in HU-treated SS relative to SS microparticle peptide MS1 chromatogram intensities that were combined for each protein into protein-level data. All peptide-spectrum matches were obtained using a strict $1 \%$ protein FDR. $P$ values were generated by applying a $t$ test statistic to log-transformed fold change values for each mouse biological condition. (D-F) Relative ion current intensity fold changes corresponding to each $\mathrm{Hb}$ amino acid-specific PTM from HU-treated and untreated SS mouse shear stress MPs $(n=3)$; measurements were determined by Hb peptide MS1 chromatogram intensities for each PTM. All peptide-spectrum matches were obtained using a strict $1 \%$ protein FDR, and $P$ values were generated by applying a $t$ test statistic for each mouse biological condition. (C) Representative Western blot showing comparative Hb binding in immunoprecipitated band 3 elute of RBC MPs from AA, SS, and SS treated with HU. Top panel: equal loading in band 3 protein input. Lower panel: presence of Hb $\alpha$ protein in the band 3 immunoprecipitate elute. (H) Average band intensities of $\mathrm{Hb}$ in band 3 immunoprecipitate from AA, SS, and SS + HU MPs were measured using Bio-Rad Image Lab software and expressed as relative density following normalization with corresponding band 3 protein bands. Bars represent average mean value; each dot in the bars represents an individual data point; and vertical error bars represent SEM. Student's $t$ test, 2 -tailed, ${ }^{*} P<0.05(n=3)$.

Most of these proteins are also seen in the MP proteome data. Interestingly, antioxidant proteins seen to be upregulated in the RBC lysate are primarily involved in iron homeostasis, including serotransferrin, glutaredoxin-3, and transferrin-like protein. We also checked the levels of some important glycolytic and MAPK enzymes in the AA and SS RBC lysates by Western blotting. Specifically, the level of ERK1/2 enzyme, but not glycolytic enzymes such as GAPDH, was found to be upregulated in the SS RBCs (data not shown).

In contrast to the volcano plots represented in Figure $8, \mathrm{~A}$ and $\mathrm{B}$, the volcano plot in Figure $8 \mathrm{C}$ representing the proteomic comparison of SS and SS HU samples indicates that most proteins were similar in abundance, with data points clustered in the center of the plot, which represents 0 -fold change (see Supplemental Table 5). While protein levels associated with oxidative stress were similar in HU-treated and untreated SS samples, there were clear differences in $\mathrm{Hb}$ PTMs which is represented by the graphs in Figure 8, D-F. Moreover, Hb PTM fold change values (Figure 8, D-F) representing $\beta$ Cys93 oxidation and $\beta$ Lys 96 and $\beta$ Lys 145 ubiquitination, were lower for HU-treated mice, suggesting that HU did provide antioxidative protection. To explore the effects of HU treatment on the interaction between RBC membrane-bound band 3 and $\mathrm{Hb}$, we conducted a co-IP assay using RBC-derived MPs from Townes- SS mice treated with or without HU. We compared band 3 immunoprecipitates from shear stress MPs obtained from AA mice, SS mice, and SS mice treated with HU (Figure 8G). Relative intensities of $\mathrm{Hb}$ bands in band 3 eluates showed a significant increase in $\mathrm{Hb}$ level in band 3 immunoprecipitate of SS MPs over corresponding AA MPs following normalization with band 3 level 
(Figure $8 \mathrm{H}$ ). In this experiment, however, the HU treatment group showed a marginally lower $\mathrm{Hb}$ level associated with band 3 than corresponding SS MPs.

$H U$ antioxidative protection involves the reduction of ferrylhemoglobin $S$ through S-nitrosylation. In addition to increasing fetal $\mathrm{Hb}(\mathrm{HbF})$ levels, $\mathrm{HU}$ may also serve as an antioxidant $(34,35)$. Therefore, we next investigated the effect of $\mathrm{HU}$ on ferryl $\mathrm{Hb}$. Figure 9A shows spectral changes reflecting $\mathrm{H}_{2} \mathrm{O}_{2}$-induced $\mathrm{Hb}$ oxidation and capturing of the ferryl species as a sulfHb intermediate upon sodium sulfide $\left(\mathrm{Na}_{2} \mathrm{~S}\right)$ addition (the resultant sulfHb absorbs strongly at $620 \mathrm{~nm}$ ). At a 1:2.5 (heme $/ \mathrm{H}_{2} \mathrm{O}_{2}$ ) ratio, there was a clear reduction in the 620 -nm absorption peak after addition of $\mathrm{HU}$, and ferryl/sulfHb levels were considerably reduced, from $27 \mu \mathrm{m}$ to $17 \mu \mathrm{m}$. The above trend is further exemplified in Figure 9B, whereby the formation of ferryl $\mathrm{Hb} / \mathrm{sulfHb}$ was increased in a dose-dependent manner with the addition of $\mathrm{H}_{2} \mathrm{O}_{2}$. Furthermore, by adding $\mathrm{HU}$, the formation of these highly reactive forms of $\mathrm{Hb}$ were clearly suppressed at all $\mathrm{H}_{2} \mathrm{O}_{2}$ /heme ratios. Overall, these findings are consistent with prior reports indicating that $\mathrm{HU}$ generates $\mathrm{NO}$ as part of its rapid decomposition and in the presence of $\mathrm{Hb}$ causes $S$-nitrosylation of Cys93 (36). Indeed, MS analysis of the peptide that contained $\beta C y s 93$ (residues 83-104) confirmed the formation of $S$-nitrosylated $\mathrm{Hb}(\mathrm{SNO})$ in the presence of $\mathrm{HU}$, as shown in Figure 9C. This may mechanistically explain the antioxidative properties of $\mathrm{HU}(34,35)$ and is also consistent with recent reports showing that reversible cysteine oxidative posttranslational modifications such as $S$-nitrosylation play a role in regulating protein structure and function (37).

\section{Discussion}

SCD RBCs are subjected to constant endogenous and exogenous oxidative stresses, which in turn increase hemolysis and contribute to vasculopathies, one of the hallmarks of this disease (5). Moreover, recent studies on SCD patient blood samples have revealed an increase in sickle RBCs retaining mitochondria when compared with samples from healthy individuals. Using an accompanying experimental SCD mouse model, the same report also indicated that excessive levels of ROS were associated with abnormal mitochondrial retention $(30,31)$.

Cumulative internal oxidative stress and a decrease in antioxidant defenses are coupled with increasing $\mathrm{Hb}$ nonenzymatic oxidation (autoxidation) (38) and pseudoperoxidase activities in the presence of oxidants (such as $\mathrm{H}_{2} \mathrm{O}_{2}$ ) in SCD (39). In fact, ferryl $\mathrm{Hb}$ and the radical byproducts of this reaction are capable of interacting with other biological and structural proteins within RBCs. Ferryl HbS has indeed been found to inhibit actin polymerization in malaria-infected $\mathrm{HbS} \mathrm{RBCs}$, in spite of the presence of RBC antioxidative enzymes (40). Compared with steady-state conditions, the occurrence of vaso-occlusive crises in SCD has been shown to be associated with an increase in the levels of both RBC-derived MPs and extracellular $\mathrm{Hb}$ in the circulation. The common byproduct resulting from free $\mathrm{Hb}$ oxidation and from $\mathrm{Hb}$-laden MPs is heme, which has been shown to initiate inflammatory responses $(3,41)$.

In the present study, we examined a potential mechanistic link between $\mathrm{Hb}$ internal oxidative pathways, membrane alteration, MP formation, and vascular toxicity in a Townes-transgenic SCD mouse model. This model is ideal for these studies because mice live in a controlled environment, possess less genetic variability, and therefore require a small sample size for acquiring statistically significant data. We monitored $\mathrm{Hb}$ oxidative changes within MPs over an extended period of time for the following reasons: (a) MPs derived from sickle RBCs have been shown to be important delivery vehicles for $\mathrm{Hb}$ and heme $(3,41)$ and (b) cellular and subcellular entrapment of RBC MPs and internalization by cells have been observed to modulate function in a time-, dose-, and temperature-dependent manner (42). Furthermore, the mechanism of $\mathrm{Hb} /$ heme loss and time frame by which heme is released from MPs are incompletely characterized.

As shown in Figure 2D, we found high levels of metHb, up to $30 \%-40 \%$ of total $\mathrm{Hb}$ in SS MPs at the start of the incubation period, which was then reduced slightly by residual antioxidant enzymes from the mother cells. This agrees with a recent photometric analysis of SS RBC-derived particles (3) but contradicts an earlier reported observation that found that $\mathrm{Hb}$ existed predominantly in the ferrous form in MP-derived samples from outdated normal blood (43). HRAM-MS analysis of the proteome at 0 and 30 hours showed a dramatic reduction in antioxidant protein levels within RBC-derived SS MPs at the end of the observation period. Catalase, SOD, glutathione peroxidase 1, and peroxiredoxin 6 were among the proteins found to be significantly impaired. The depletion of this cytosolic reductive capacity left these $\mathrm{Hb}$ oxidative reactions unregulated and consequently resulted in perpetuating oxidative changes and the formation of damaging ferryl species. These self-inflected oxidative changes (including the formation of the ferryl heme 

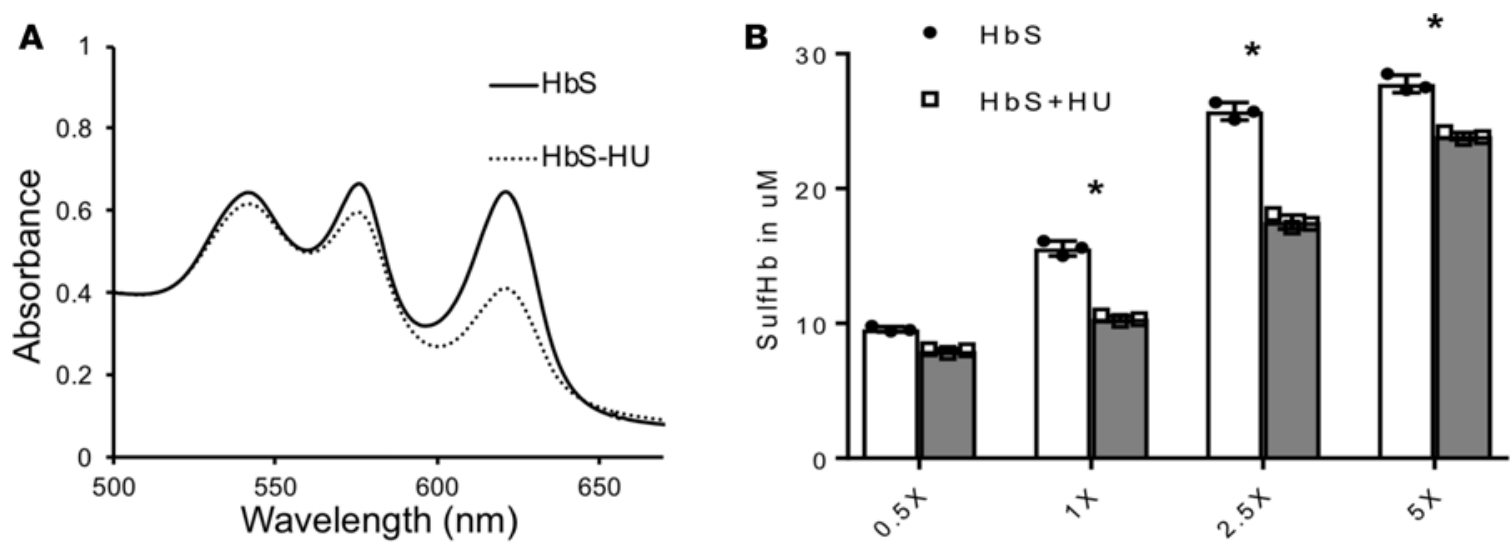

$\mathrm{H}_{2} \mathrm{O}_{2} / \mathrm{HbS}(\mathrm{mol} / \mathrm{mol})$

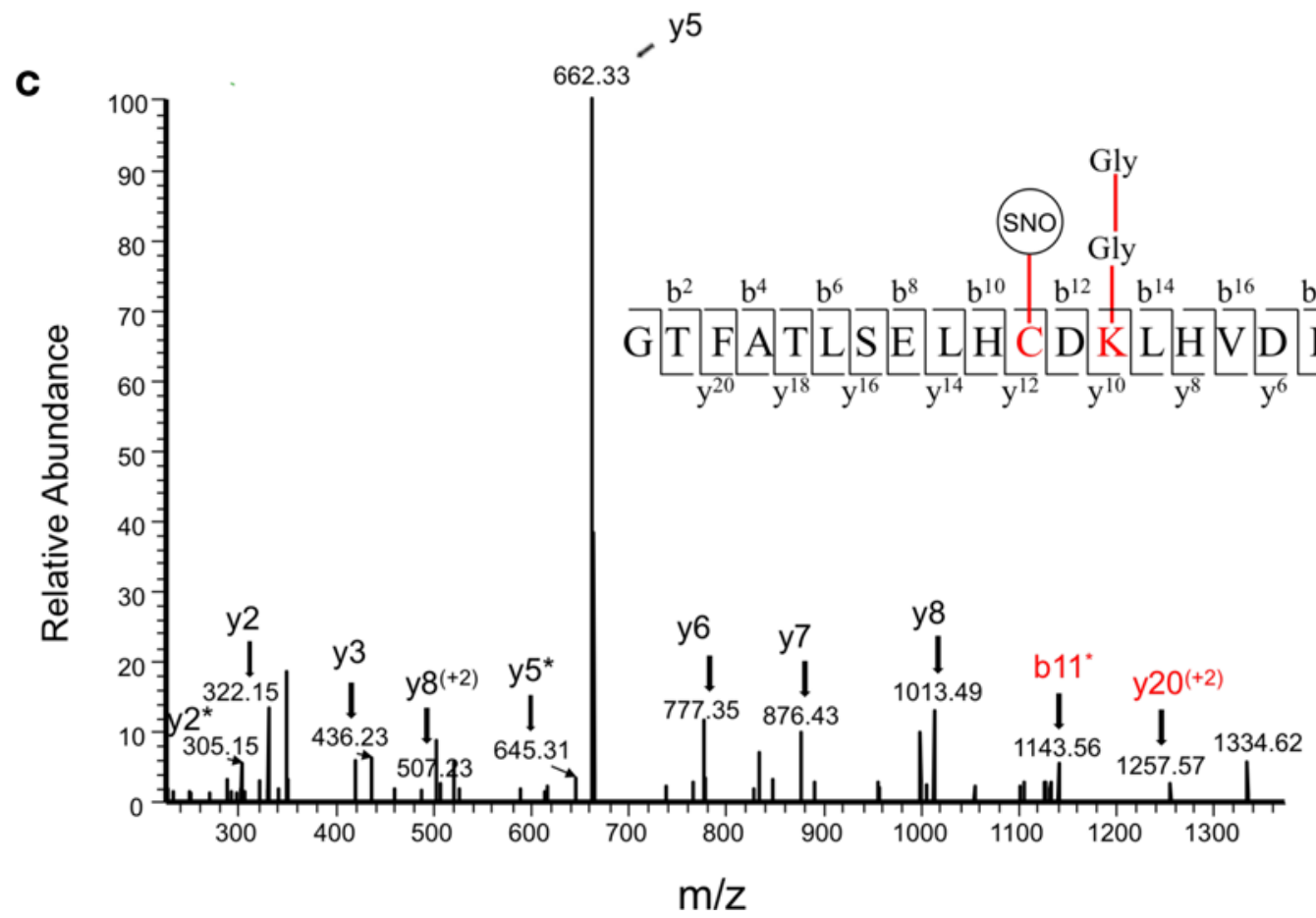

Figure 9. Hydroxyurea reduces ferryl heme and shields $\beta$ Cy93 by a process of $\boldsymbol{S}$-nitrosylation. Effects of $\mathrm{HU}$ on the levels of ferryl $\mathrm{Hb}$ (made with the addition of $150 \mu \mathrm{M} \mathrm{H}_{2} \mathrm{O}_{2}$ ). (A) Overlaid sulfHb spectra (absorption at $620 \mathrm{~nm}$ ) of HbS (60 $\mu \mathrm{M}$ ) treated (dashed line) and non-treated with HU (240 $\left.\mu \mathrm{M}\right)$ (solid line). (B) Amounts of sulfHb produced at various ratios of $\mathrm{H}_{2} \mathrm{O}_{2}$ to $\mathrm{Hb}$; nontreated $\mathrm{HbS}$ (solid black) and HU-treated (pattern). (C) MS/MS fragmentation spectrum representing the $y$ and $b$ ions matched to the modified tryptic peptide GTFATSELHCDKLHVDPENFR (residues 83-104) (residues 82-104) from Hb incubated with $\mathrm{HU}$; this peptide contains the diglycine signature modification (at $\beta$ Lys96) associated with ubiquitination and S-nitrosylation (at $\beta$ Cys93). Bars represent average mean value; each dot in the bars represents an individual data point, and vertical error bars represent SEM. Student's $t$ test, 2-tailed, ${ }^{*} P<0.05 \mathrm{HbS}$ vs. corresponding HbS $+\mathrm{HU}(n=3)$.

and its radical result in $\mathrm{Hb} \beta$ alteration) can be seen in our HPLC profiles of $\mathrm{Hb}$ in the SS MPs. In addition to this work showing MP proteomic differences with respect to antioxidant enzymes (which reflects change in pathophysiology of $\mathrm{HbS}$ mice), recent metabolic profiling of normal and SCD human RBC samples has also revealed the impact of $\mathrm{HbS}$ on the metabolome. Specifically, Darghouth et al. recently reported that SCD patients exhibit changes to oxidative damage control systems; in SCD RBCs, glutathione and oxidized glutathione levels were decreased, whereas the concentrations of their precursors as well as ascorbate metabolism intermediates were increased (44).

The mouse MP proteomic landscape analysis from 2 separate comparative studies confirmed that SS mice experience HbS-induced oxidative stress. A large number of proteins previously reported to be associated with oxidative stress were significantly upregulated in the SS MP proteome (relative to the AA MPs). 
In particular, we observed a substantial increase in mitochondrial proteins. This supports a recent study that linked oxidative stress within SCD RBCs with the retention of mitochondria (30). However, TownesSS mouse blood has been reported to contain 50\%-70\% more reticulocytes and immature nucleated $\mathrm{RBCs}$ (32) compared with AA controls; the source of mitochondrial proteins (and other organellar proteins) in our mass spectrometric analysis of the mouse proteome (see Supplemental Tables 1-5) could therefore be partially due to the presence of reticulocytes. In addition to changes in the levels of antioxidant enzymes, we also observed increases in translation factors, molecular chaperones, UPS proteins, and those associated with the 20S proteasome - all of which are consistently associated with oxidative stress (45-47).

Additionally, $\mathrm{HbS}$ was significantly more oxidized at the hotspot residue $\beta \mathrm{Cys} 93$ relative to $\mathrm{HbA}$ from AA mice. This further supports the oxidation kinetics data (described herein) indicating that SS MPs contain higher levels of ROS. Generally, when naturally occurring mutant Hbs (including $\mathrm{HbS}$ ) are chemically challenged with $\mathrm{H}_{2} \mathrm{O}_{2}$, extensive globin chain crosslinking and irreversible modification of key $\beta$ subunit amino acids (known as oxidation hotspots) occurs $(13,48)$. These amino acids ( $\beta$ His $146, \beta$ Tyr 145 , $\beta$ Cys93, $\beta$ Trp37, and $\beta$ Pro36) are located in proximity to the $\alpha / \beta$ interface, which happens to be the point of contact with the antioxidant $\mathrm{Hp}(8,49)$. This is consistent with our earlier observations that $\beta$ Cys 93 and other hotspot residues can be considered as a reliable reporter for oxidative modifications and degradation for the $\mathrm{Hb}$ molecule $(13,25)$.

We also identified HbS PTMs such as ubiquitination at K96 and K145. These ubiquitinated sites in $\mathrm{HbS}$ were higher in all SS MPs examined in this report (see Supplemental Tables 1-3 and 6). Ubiquitination is an ATP-dependent modification requiring the UPS machinery (selective proteolytic system in eukaryotic systems), which has recently been reported to be functionally active in mature normal RBCs $(47,50)$. Interestingly, ubiquitinated protein targeting by the UPS has also been found to be associated with protein oxidation and subsequent aggregation (24). Our method detects the diglycine moiety that is associated with ubiquitination but does not differentiate between mono- and polyubiquitination (51). However, orthogonal co-IP experiments following inhibition of the proteasomal system and deubiquitinase enzymes present in RBCs by MG132 and NEM, respectively, confirmed the existence of ubiquitinated forms of $\mathrm{Hb}$, potentially signaling an accumulation of modified $\mathrm{Hb}$ that can lead to its degradation. Further, Townes-SS mice have a population of immature RBCs, so it would not be surprising to see an accumulation of many of newly synthesized ubiquitinated $\mathrm{Hbs}(33,52)$. As we consistently found higher levels of Hb ubiquitination and $\mathrm{Hb}$ oxidation in all SS RBC-derived MPs, the most likely possibility is impairment of the proteasomal machinery in SCD due to a redox imbalance leading to a buildup of ubiquitinated (mono-, di-, or poly-) $\mathrm{Hb}$. By suggesting a link between $\mathrm{HbS}$ oxidation and ubiquitination, our MP proteome data provide insight into a potential mechanism of removing toxic $\mathrm{HbS}$ (during oxidative stress). Our data also reveal a possible link between ubiquitination and the DAMP molecular system, since it has recently been shown that the TLR4 signaling pathways are also regulated by the ubiquitin-proteasome system (53).

$\mathrm{Hb}$ oxidation products (including hemichromes) have high affinity toward the cytoplasmic domain of band 3 and are reported to mediate oxidative crosslinking through disulfide bonds $(28,29,54)$. Band 3 clustering has been shown to be associated with blebbing and MP generation, which are important characteristics of SCD and $\beta$-thalassemias $(55,56)$. In this study, we used purified ferryl $\mathrm{Hb}$ (by reacting $\mathrm{H}_{2} \mathrm{O}_{2}$ with metHb and rapidly removing the oxidant by chromatography) in a medium of ghost cells (57). We also avoided the use of phenylhydrazine, a commonly utilized oxidant known to generate redox-inactive hemichromes, in which the heme iron is hexa-coordinately bound to the protein (58). Using specific antibodies against band 3 and $\mathrm{Hb}$, we were able to demonstrate that ferryl $\mathrm{Hb}$ is more effective in producing complexes with band 3 than both the ferric and ferrous forms of $\mathrm{Hb}$. Co-IP followed by MS confirmed the presence of membrane-associated proteins in the medium, to which ferryl $\mathrm{Hb}$ was added. This is consistent with recent experiments in which ghost cells derived from normal human RBCs were used (57). Taken together, these experiments showed that ferryl $\mathrm{Hb}$ (but not hemichromes) induces more membrane complexes and that approximately $50 \%$ of this binding involves reactive sulfhydryl groups, likely from $\beta$ Cys 93 (57).

Our experiments with endothelial cells further indicated that SS MP toxicities are related to their distinct oxidative profile. Camus et al. previously showed heme accumulation and $\mathrm{Hb}$ within endothelial cells (following adhesion of sickle RBC-derived MPs) resulted in rapid generation of intracellular ROS and activation of apoptosis (3). $\mathrm{Hb}$ accumulation within tubulointerstitial or intraglomerular capillaries has also been shown by the same group in human SCD renal biopsies (16). Selective passage of blood or plasma components into the adjacent tissues is well controlled by vascular endothelium acting as a dynamic barrier. Various stress conditions 
including inflammatory mediators are known to damage vascular endothelial integrity (59). We have previously shown that $\mathrm{HbS}$ (especially in its oxidized form) can damage endothelial monolayer integrity more effectively than $\mathrm{HbA}(60)$. Our results showing a loss of monolayer integrity by SS MPs may be attributable to their high $\mathrm{HbS}$ content coupled with higher rates of oxidation of $\mathrm{HbS}$ over $\mathrm{HbA}$, and the formation of more persistent and damaging oxidized species that can target other biological molecules (13). Our data establish a link between the loss of endothelial monolayer integrity with concomitant apoptosis and induction of HO-1 expression by SS MPs. We therefore measured HO-1 as secondary evidence of $\mathrm{Hb}$ - (and heme-) mediated toxicity in HUVECs. Higher HO-1 expression in SS MP-treated HUVECs is indicative of increased $\mathrm{Hb}$ oxidation and catabolism, consistent with those previous reports (3). Moreover, our results also indicated an impairment in mitochondrial bioenergetics, noticeable specifically under an FCCP-induced uncoupled state. The loss in mitochondrial reserve capacity in endothelial cells by SS MPs indicates a failure of the mitochondrial population to respire to their maximum possible rate during high energetic demand. We and others have previously shown that HO-1 expression with its localization into mitochondria leads to a compromised bioenergetic state under the influence of oxidized $\mathrm{Hb}$ or heme $(61,62)$. Therefore, it is possible that a similar mechanism is also operational in our MP-based model. However, the major metabolic pathway in endothelial cells, i.e., glycolysis, remains unaltered by MP exposure. It was previously demonstrated that erythrocyte MPs can trigger rapid vaso-occlusions in tissues sensitive to ischemic injury (16). Our present observations with HUVECs exposed to SS MPs suggest that HbS-rich MPs may cause significant pathophysiological impairment as seen in SCD and other hemolytic disorders, including decreased vasodilation and massive vaso-occlusion, by damaging vascular integrity.

To investigate the impact of HU treatment on oxidative pathways of $\mathrm{Hb}$ in both RBCs and MPs, we analyzed blood from untreated mice and those treated with HU; overall, the abundance levels of most proteins were similar for both mouse populations. However, we observed reductions in $\mathrm{HbS}$ $\beta$ Cys93 and ubiquitination of $\beta$ Lys 96 as well as $\beta$ Lys145 relative to untreated SS mice, which suggests an additional antioxidant benefit from HU (63). This is also in keeping with recent clinical trials on HU treatment of SCD children, in which children were reported to show decreases in plasma MPs derived from RBCs and platelets (64).

In summary, $\mathrm{Hb}$ transformation to higher oxidative forms was markedly increased in MPs generated from SCD mice in a pattern similar to that of their parent RBCs (see the scheme in Figure 10). Ferryl $\mathrm{Hb}$ (once formed) manifested several oxidative changes, including irreversible oxidation of $\beta$ Cys 93 and ubiquitination of $\beta$ Lys96 and $\beta$ Lys145. Ferryl $\mathrm{Hb}$ also oxidatively targeted band 3, which resulted in band 3-mediated complex formation (with $\mathrm{Hb}$ and other membrane proteins) and activation of the UPS pathway. HU appeared to have an antioxidant effect, as it potentially minimized the consequences of these processes through $S$-nitrosylation of $\beta C y$ s93. When MPs were incubated with endothelial cells, this led to mitochondrial dysfunction and eventually apoptotic cell death. Taken together, these mechanistic results suggest that $\mathrm{Hb}$ oxidative pathways drive oxidative changes within SCD RBCs and similarly in RBC-derived MPs and that antioxidative interventions may slow down and/or prevent $\mathrm{Hb}$-oxidative side reactions in SCD.

\section{Methods}

Sickle cell mouse model and treatment with HU. HbSS-Townes mice were created by knocking in human $\alpha$ and $\beta^{S}$ globins into the sites where murine $\alpha$ and $\beta$ globins were knocked out. HbSS-Townes mice have severe anemia and an RBC half-life of 2.5 days. HbAA-Townes control mice (RBC half-life, 16 days) were created by replacing $\beta^{S}$ with $\beta^{\text {A. }}$ (65). Heparinized blood was obtained from Townes mice (total 21 Townes-AA and Townes-21 SS). In some experiments, Townes-SS mice were treated with HU for 10 days with a diet containing $100 \mathrm{mg} / \mathrm{kg} \mathrm{HU}$ in water/methylcellulose, while control animals had water/methylcellulose vehicle only. Both groups drank the same volume of water over the 10 days. Isoelectric focusing analysis of different mouse blood samples was carried out using RBC lysates. See Supplemental Methods for details.

Preparation of MPs from mouse blood. For the majority of experiments, MPs were generated by shear stress from RBCs (18). Heparinized whole blood was used for the preparation of RBC MPs. Shear stress MPs were generated by passing PBS-washed RBCs rapidly through a $25 \mathrm{G}$ needle. A detail description of $\mathrm{RBC}$-derived and circulating MP preparation is provided in Supplemental Methods.

Flow cytometric and electron microscopic analysis of RBC MPs. RBC-derived MPs were counted by flow cytometry and visualized through cryogenic transmission electron microscopy (cryo-TEM). For details, see Supplemental Methods. 


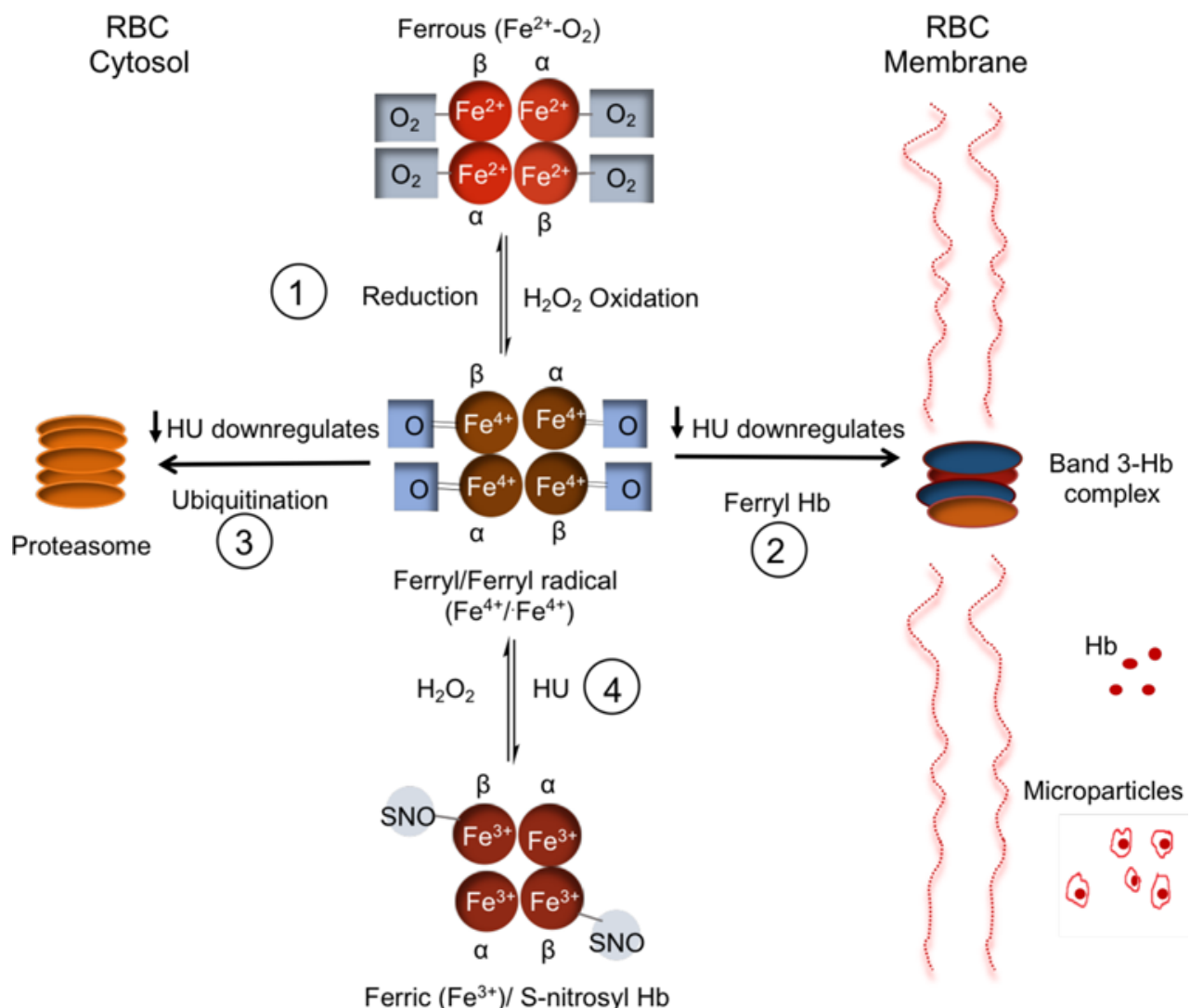

Figure 10. Proposed erythrocyte internal oxidative changes that trigger membrane alternations and microparticle formation. Oxidative reactions within RBCs include the following pathways: (1) Ferrous $\mathrm{Hb}\left(\mathrm{Fe}^{2+}\right)$ undergoes spontaneous oxidation (autoxidation) to form ferric $\mathrm{Hb}\left(\mathrm{Fe}^{3+}\right)$, and in the presence of $\mathrm{H}_{2} \mathrm{O}_{2}$ ferryl $\mathrm{Hb}\left(\mathrm{Fe}^{4+}\right.$ ) is formed when antioxidative mechanisms are compromised. Ferryl $\mathrm{Hb}$ undergoes oxidative changes including irreversible oxidation of $\beta$ Суs 93 and ubiquitination of some lysine residues. Ferryl $\mathrm{Hb}$ can oxidatively target band 3 , leading to complex formation and microparticle formation (2) or denaturation and proteasome degradation (3). Some ferryl Hbs revert back to ferric by a process of autoreduction (4). Hydroxyurea acts as an antioxidant by forming SNO-Hb at $\beta$ Cys 93 by a process of $S$-nitrosylation, thereby minimizing the consequences of these processes (adapted with modification from ref. 23).

Autoxidation experiments. Quantification and determination of the oxidation state within MPs were achieved by using a Nanodrop 1000 spectrophotometer together with conventional spectrophotometers. Multicomponent analysis was used to calculate ferrous (oxy-), met-, and ferryl $\mathrm{Hb}$ based on the extinction coefficients of each species (22). For further details and biochemical analysis of MPs, see Supplemental Methods.

Reverse-phase HPLC analyses of Hb oxidative changes within MP oxidation. Reverse-phase HPLC analyses of MPs were performed on a Zorbax 300 SB C3 column $(4.6 \times 250 \mathrm{~mm})$ using a Waters HPLC system as described earlier (13). For details, see Supplemental Methods.

Preparation of ferric and ferryl Hbs. Ferric (met) and ferryl $\mathrm{Hb}$ were generated as previously reported (13, 22). See Supplemental Methods for details.

Hydrogen peroxide-mediated oxidation of $\mathrm{Hb}$ and the effects of $\mathrm{HU}$. Dose-dependent $\mathrm{H}_{2} \mathrm{O}_{2}$-induced ferryl $\mathrm{Hb}$ species formation was evaluated with and without HU following previously described methods (22). For verification of the ferryl intermediate, $2 \mathrm{mM} \mathrm{Na}_{2} \mathrm{~S}$ was added immediately to derivatize the ferryl heme into sulfHb (66). See Supplemental Methods for details.

Western blotting and co-IP. Immunoblotting was done as previously reported (62). A detail description is provided in Supplement Methods.

Vascular endothelial cell culture and treatments. Cryopreserved HUVECs were cultured using M200 media according to the supplier's protocol (Thermo Fisher Scientific).

The cells were exposed to different RBC-derived MPs isolated from Townes mouse blood $(1,000$ $\mathrm{MPs} / \mu \mathrm{l}$ ) for varying periods of time up to 12 hours. See Supplement Methods for details. 
Assessment of endothelial damage by Transwell assay and apoptotic activation. Loss of endothelial monolayer integrity was studied by monitoring passage of FITC-labeled dextran $(40 \mathrm{kDa})$ through an HUVEC monolayer grown on collagen-coated PTFE-Transwell membrane inserts (Corning Inc.) as described previously (60). HUVEC monolayer integrity was also observed by fluorescence microscopy using an antibody against endothelium-specific VE-cadherin. The percent apoptotic cell population in MP-challenged HUVECs was determined by a Nexcelom Automated Cell Counter (Nexcelom Bioscience). More details are provided in the Supplemental Methods.

Mitochondrial bioenergetic and glycolytic flux measurements with Seahorse analyzer. Endothelial OCR and ECAR were measured to assess mitochondrial respiration and glycolysis, respectively, and plotted using an Agilent Seahorse XF24 extracellular flux analyzer, as per the manufacturer's instructions and as described previously $(13,60)$. Various cellular bioenergetic parameters were calculated from average rate data obtained from individual wells as described previously $(67,68)$. See Supplemental Methods for more details.

$L C-M S$ analysis of band 3 co-IP. Proteins in band 3 immunoprecipitates resolved by SDS-PAGE were subjected to MS analysis using LC-MS. Gel slices were excised, digested, and then analyzed by MS. See Supplemental Methods for more details.

Proteomic analysis of MPs. Prior to MS analysis, the total protein concentration of all RBC-derived MPs was determined using a standard BCA assay. Afterward, samples were tryptically digested, desalted, and analyzed by MS as previously described (69). Quantitative data processing and visualization were performed using both Protalizer DDA software (Vulcan Analytical) version 1.1.2418 and X! Tandem Piledriver search engine version 2015.04.01.1 (70, 71). See Supplemental Methods for further details.

Statistics. See Supplemental Methods for a detailed description of statistical analysis.

Study approval. All mice were maintained at the University of Minnesota Medical School. All animal studies were approved by the University of Minnesota Institutional Animal Care and Use Committee.

\section{Author contributions}

SJ and AIA conceived and designed the study. SJ and MBS performed a majority of the experiments and data analysis. FM, TK, and WH performed other biochemical experiments and data analysis. SDP and IT performed electron microscopic studies and flow cytometry. MRH assisted in proteomic data analysis. GMV and JDB contributed to the overall design of animal experiments and manuscript editing. JS contributed to the development of the MP preparation protocol and the design of physical characterization studies of MPs. SJ, MBS, and AIA drafted the manuscript. All authors read and approved the final manuscript.

\section{Acknowledgments}

This work was supported by the NIH/NHLBI under grants HL110900 (to AIA) and by the US Food and Drug Administration (MODSCI 2012 to AIA). JDB and GMV receive research funding from the NIH (R01HL114567).

Address correspondence to: Abdu I. Alayash, Laboratory of Biochemistry and Vascular Biology, Center for Biologics Evaluation and Research, Food and Drug Administration, 10903 New Hampshire Avenue, Building 52/72, Room 4106, Silver Spring, Maryland 20993, USA. Phone: 240.402.9350; Email: abdu.alayash@fda.hhs.gov.

1. Hebbel RP. Ischemia-reperfusion injury in sickle cell anemia: relationship to acute chest syndrome, endothelial dysfunction, arterial vasculopathy, and inflammatory pain. Hematol Oncol Clin North Am. 2014;28(2):181-198.

2. Schaer DJ, Buehler PW, Alayash AI, Belcher JD, Vercellotti GM. Hemolysis and free hemoglobin revisited: exploring hemoglobin and hemin scavengers as a novel class of therapeutic proteins. Blood. 2013;121(8):1276-1284.

3. Camus SM, et al. Circulating cell membrane microparticles transfer heme to endothelial cells and trigger vasoocclusions in sickle cell disease. Blood. 2015;125(24):3805-3814.

4. Gizi A, et al. Assessment of oxidative stress in patients with sickle cell disease: the glutathione system and the oxidant-antioxidant status. Blood Cells Mol Dis. 2011;46(3):220-225.

5. Chirico EN, Pialoux V. Role of oxidative stress in the pathogenesis of sickle cell disease. IUBMB Life. 2012;64(1):72-80.

6. Voskou S, Aslan M, Fanis P, Phylactides M, Kleanthous M. Oxidative stress in $\beta$-thalassaemia and sickle cell disease. Redox Biol. 2015;6:226-239.

7. George A, et al. Erythrocyte NADPH oxidase activity modulated by Rac GTPases, PKC, and plasma cytokines contributes to oxidative stress in sickle cell disease. Blood. 2013;121(11):2099-2107. 
8. Belcher JD, et al. Heme triggers TLR4 signaling leading to endothelial cell activation and vaso-occlusion in murine sickle cell disease. Blood. 2014;123(3):377-390.

9. Baek JH, et al. Hemoglobin-driven pathophysiology is an in vivo consequence of the red blood cell storage lesion that can be attenuated in guinea pigs by haptoglobin therapy. J Clin Invest. 2012;122(4):1444-1458.

10. Rentsendorj O, Zhang X, Williams MC, Buehler PW, D’Agnillo F. Transcriptional suppression of renal antioxidant enzyme systems in guinea pigs exposed to polymerized cell-free hemoglobin. Toxics. 2016;4(1):E6.

11. Ghosh S, et al. Extracellular hemin crisis triggers acute chest syndrome in sickle mice. J Clin Invest. 2013;123(11):4809-4820

12. Alayash AI. Hemoglobin-based blood substitutes and the treatment of sickle cell disease: more harm than help? Biomolecules. $2017 ; 7(1)$.

13. Kassa T, et al. Sickle cell hemoglobin in the ferryl state promotes $\beta$ Cys-93 oxidation and mitochondrial dysfunction in epithelia lung cells (E10). J Biol Chem. 2015;290(46):27939-27958.

14. Hebbel RP, Key NS. Microparticles in sickle cell anaemia: promise and pitfalls. Br J Haematol. 2016;174(1):16-29.

15. Said AS, Doctor A. Influence of red blood cell-derived microparticles upon vasoregulation. Blood Transfus. 2017;15(6):522-534.

16. Camus SM, et al. Erythrocyte microparticles can induce kidney vaso-occlusions in a murine model of sickle cell disease. Blood. 2012;120(25):5050-5058.

17. Niihara Y, Zerez CR, Akiyama DS, Tanaka KR. Increased red cell glutamine availability in sickle cell anemia: demonstration of increased active transport, affinity, and increased glutamate level in intact red cells. J Lab Clin Med. 1997;130(1):83-90.

18. Jy W, Johansen ME, Bidot C Jr, Horstman LL, Ahn YS. Red cell-derived microparticles (RMP) as haemostatic agent. Thromb Haemost. 2013;110(4):751-760.

19. Osawa Y, Darbyshire JF, Meyer CA, Alayash AI. Differential susceptibilities of the prosthetic heme of hemoglobin-based red cell substitutes. Implications in the design of safer agents. Biochem Pharmacol. 1993;46(12):2299-2305.

20. Reeder BJ, Sharpe MA, Kay AD, Kerr M, Moore K, Wilson MT. Toxicity of myoglobin and haemoglobin: oxidative stress in patients with rhabdomyolysis and subarachnoid haemorrhage. Biochem Soc Trans. 2002;30(4):745-748.

21. Boutaud $\mathrm{O}$, et al. Acetaminophen inhibits hemoprotein-catalyzed lipid peroxidation and attenuates rhabdomyolysis-induced renal failure. Proc Natl Acad Sci U S A. 2010;107(6):2699-2704.

22. Meng F, Alayash AI. Determination of extinction coefficients of human hemoglobin in various redox states. Anal Biochem. 2017;521:11-19.

23. Alayash AI. Oxidative pathways in the sickle cell and beyond. Blood Cells Mol Dis. 2018;70:78-86.

24. Tramutola A, et al. Polyubiquitinylation profile in down syndrome brain before and after the development of Alzheimer neuropathology. Antioxid Redox Signal. 2017;26(7):280-298.

25. Kassa T, Strader MB, Nakagawa A, Zapol WM, Alayash AI. Targeting $\beta$ Cys93 in hemoglobin S with an antisickling agent possessing dual allosteric and antioxidant effects. Metallomics. 2017;9(9):1260-1270.

26. Strader MB, Alayash AI. Exploring oxidative reactions in hemoglobin variants using mass spectrometry: lessons for engineering oxidatively stable oxygen therapeutics. Antioxid Redox Signal. 2017;26(14):777-793.

27. Terra HT, Saad MJ, Carvalho CR, Vicentin DL, Costa FF, Saad ST. Increased tyrosine phosphorylation of band 3 in hemoglobinopathies. Am J Hematol. 1998;58(3):224-230.

28. Walder JA, et al. The interaction of hemoglobin with the cytoplasmic domain of band 3 of the human erythrocyte membrane. $J$ Biol Chem. 1984;259(16):10238-10246.

29. Waugh SM, Low PS. Hemichrome binding to band 3: nucleation of Heinz bodies on the erythrocyte membrane. Biochemistry. $1985 ; 24(1): 34-39$

30. Jagadeeswaran R, et al. Pharmacological inhibition of LSD1 and mTOR reduces mitochondrial retention and associated ROS levels in the red blood cells of sickle cell disease. Exp Hematol. 2017;50:46-52.

31. Jagadeeswaran R, Rivers A. Evolving treatment paradigms in sickle cell disease. Hematology Am Soc Hematol Educ Program. 2017;2017(1):440-446

32. Ryan TM, Ciavatta DJ, Townes TM. Knockout-transgenic mouse model of sickle cell disease. Science. 1997;278(5339):873-876.

33. Belcher JD, et al. Control of oxidative stress and inflammation in sickle cell disease with the Nrf2 activator dimethyl fumarate. Antioxid Redox Signal. 2017;26(14):748-762.

34. Agil A, Sadrzadeh SM. Hydroxy-urea protects erythrocytes against oxidative damage. Redox Rep. 2000;5(1):29-34

35. Torres Lde S, et al. The influence of hydroxyurea on oxidative stress in sickle cell anemia. Rev Bras Hematol Hemoter. 2012;34(6):421-425.

36. Huang J, Zou Z, Kim-Shapiro DB, Ballas SK, King SB. Hydroxyurea analogues as kinetic and mechanistic probes of the nitric oxide producing reactions of hydroxyurea and oxyhemoglobin. J Med Chem. 2003;46(17):3748-3753.

37. Wang SB, Murray CI, Chung HS, Van Eyk JE. Redox regulation of mitochondrial ATP synthase. Trends Cardiovasc Med. 2013;23(1):14-18

38. Hebbel RP, Ney PA, Foker W. Autoxidation, dehydration, and adhesivity may be related abnormalities of sickle erythrocytes. Am J Physiol. 1989;256(3 pt 1):C579-C583.

39. Alayash AI. Oxygen therapeutics: can we tame haemoglobin? Nat Rev Drug Discov. 2004;3(2):152-159.

40. Cyrklaff M, et al. Hemoglobins S and C interfere with actin remodeling in Plasmodium falciparum-infected erythrocytes. Science. 2011;334(6060):1283-1286.

41. Vercellotti GM. Special delivery: microparticles convey heme. Blood. 2015;125(24):3677-3678.

42. Schneider DJ, Speth JM, Penke LR, Wettlaufer SH, Swanson JA, Peters-Golden M. Mechanisms and modulation of microvesicle uptake in a model of alveolar cell communication. J Biol Chem. 2017;292(51):20897-20910.

43. Donadee $\mathrm{C}$, et al. Nitric oxide scavenging by red blood cell microparticles and cell-free hemoglobin as a mechanism for the red cell storage lesion. Circulation. 2011;124(4):465-476.

44. Darghouth D, et al. Pathophysiology of sickle cell disease is mirrored by the red blood cell metabolome. Blood. 2011;117(6):e57-e66.

45. Fulda S, Gorman AM, Hori O, Samali A. Cellular stress responses: cell survival and cell death. Int J Cell Biol. 2010;2010:214074

46. Dantuma NP, Bott LC. The ubiquitin-proteasome system in neurodegenerative diseases: precipitating factor, yet part of the solution. Front Mol Neurosci. 2014;7:70. 
47. Neelam S, Kakhniashvili DG, Wilkens S, Levene SD, Goodman SR. Functional 20S proteasomes in mature human red blood cells. Exp Biol Med (Maywood). 2011;236(5):580-591.

48. Jia Y, Buehler PW, Boykins RA, Venable RM, Alayash AI. Structural basis of peroxide-mediated changes in human hemoglobin: a novel oxidative pathway. J Biol Chem. 2007;282(7):4894-4907.

49. Andersen CB, et al. Structure of the haptoglobin-haemoglobin complex. Nature. 2012;489(7416):456-459.

50. Vilchez D, Saez I, Dillin A. The role of protein clearance mechanisms in organismal ageing and age-related diseases. Nat Commun. 2014;5:5659.

51. Xu G, Paige JS, Jaffrey SR. Global analysis of lysine ubiquitination by ubiquitin remnant immunoaffinity profiling. Nat Biotechnol. 2010;28(8):868-873.

52. Raviv O, Heller H, Hershko A. Alterations in components of the ubiquitin-protein ligase system following maturation of reticulocytes to erythrocytes. Biochem Biophys Res Commun. 1987;145(2):658-665.

53. Lopez-Castejon G, Edelmann MJ. Deubiquitinases: novel therapeutic targets in immune surveillance? Mediators Inflamm. 2016;2016:3481371.

54. Arashiki N, Kimata N, Manno S, Mohandas N, Takakuwa Y. Membrane peroxidation and methemoglobin formation are both necessary for band 3 clustering: mechanistic insights into human erythrocyte senescence. Biochemistry. 2013;52(34):5760-5769

55. Mannu F, et al. Role of hemichrome binding to erythrocyte membrane in the generation of band-3 alterations in beta-thalassemia intermedia erythrocytes. Blood. 1995;86(5):2014-2020.

56. Ferru E, et al. Thalassemic erythrocytes release microparticles loaded with hemichromes by redox activation of p72Syk kinase Haematologica. 2014;99(3):570-578

57. Welbourn EM, Wilson MT, Yusof A, Metodiev MV, Cooper CE. The mechanism of formation, structure and physiological relevance of covalent hemoglobin attachment to the erythrocyte membrane. Free Radic Biol Med. 2017;103:95-106.

58. Mollan TL, et al. $\alpha$-Hemoglobin stabilizing protein (AHSP) markedly decreases the redox potential and reactivity of $\alpha$-subunits of human HbA with hydrogen peroxide. J Biol Chem. 2013;288(6):4288-4298.

59. Nooteboom A, Hendriks T, Ottehöller I, van der Linden CJ. Permeability characteristics of human endothelial monolayers seeded on different extracellular matrix proteins. Mediators Inflamm. 2000;9(5):235-241.

60. Jana S, Meng F, Hirsch RE, Friedman JM, Alayash AI. Oxidized mutant human hemoglobins S and E induce oxidative stress and bioenergetic dysfunction in human pulmonary endothelial cells. Front Physiol. 2017;8:1082.

61. Higdon AN, et al. Hemin causes mitochondrial dysfunction in endothelial cells through promoting lipid peroxidation: the protective role of autophagy. Am J Physiol Heart Circ Physiol. 2012;302(7):H1394-H1409.

62. Chintagari NR, Jana S, Alayash AI. Oxidized ferric and ferryl forms of hemoglobin trigger mitochondrial dysfunction and injury in alveolar type I cells. Am J Respir Cell Mol Biol. 2016;55(2):288-298.

63. Ferrone FA. Sickle cell disease: its molecular mechanism and the one drug that treats it. Int J Biol Macromol. 2016;93(Pt A):1168-1173

64. Nébor D, et al. Fetal hemoglobin and hydroxycarbamide moduate both plasma concentration and cellular origin of circulating microparticles in sickle cell anemia children. Haematologica. 2013;98(6):862-867.

65. Belcher JD, Nath KA, Vercellotti GM. Vasculotoxic and proinflammatory effects of plasma heme: cell signaling and cytoprotective responses. ISRN Oxidative Med. 2013;831596.

66. Berzofsky JA, Peisach J, Blumberg WE. Sulfheme proteins. I. Optical and magnetic properties of sulfmyoglobin and its derivatives. J Biol Chem. 1971;246(10):3367-3377.

67. TeSlaa T, Teitell MA. Techniques to monitor glycolysis. Meth Enzymol. 2014;542:91-114.

68. Dranka BP, Hill BG, Darley-Usmar VM. Mitochondrial reserve capacity in endothelial cells: The impact of nitric oxide and reactive oxygen species. Free Radic Biol Med. 2010;48(7):905-914.

69. Strader MB, et al. Post-translational transformation of methionine to aspartate is catalyzed by heme iron and driven by peroxide: a novel subunit-specific mechanism in hemoglobin. J Biol Chem. 2014;289(32):22342-22357.

70. Heaven MR, et al. Systematic evaluation of data-independent acquisition for sensitive and reproducible proteomics-a prototype design for a single injection assay. J Mass Spectrom. 2016;51(1):1-11.

71. Craig R, Beavis RC. TANDEM: matching proteins with tandem mass spectra. Bioinformatics. 2004;20(9):1466-1467. 